\title{
Order of wetting transitions in electrolyte solutions
}

\author{
Ingrid Ibagon Markus Bier 0 and S. Dietrich \\ Max-Planck-Institut für Intelligente Systeme, Heisenbergstr. 3, \\ 70569 Stuttgart, Germany and IV. Institut für Theoretische Physik, \\ Universität Stuttgart, Pfaffenwaldring 57, 70569 Stuttgart, Germany
}

(Dated: July 19, 2018)

\begin{abstract}
For wetting films in dilute electrolyte solutions close to charged walls we present analytic expressions for their effective interface potentials. The analysis of these expressions renders the conditions under which corresponding wetting transitions can be first- or second-order. Within mean field theory we consider two models, one with short- and one with long-ranged solvent-solvent and solvent-wall interactions. The analytic results reveal in a transparent way that wetting transitions in electrolyte solutions, which occur far away from their critical point (i.e., the bulk correlation length is less than half of the Debye length) are always first-order if the solvent-solvent and solvent-wall interactions are short-ranged. In contrast, wetting transitions close to the bulk critical point of the solvent (i.e., the bulk correlation length is larger than the Debye length) exhibit the same wetting behavior as the pure, i.e., salt-free, solvent. If the salt-free solvent is governed by long-ranged solvent-solvent as well as long-ranged solvent-wall interactions and exhibits critical wetting, adding salt can cause the occurrence of an ion-induced first-order thin-thick transition which precedes the subsequent continuous wetting as for the salt-free solvent.
\end{abstract}

\section{INTRODUCTION}

Recent theoretical studies of wetting phenomena in electrolyte solutions near charged walls have focused on analyzing the influence of salt and surface charge density on the wetting behavior of solvents [1 [5]. The corresponding models share certain common features such as the short range of the underlying non-electrostatic interaction potentials and the mean-field character of the approaches. The model studied in Refs. [1, 2] combines Cahn's phenomenological theory for the solvent with the Poisson-Boltzmann theory for the ions. Within this model, ions and solvent molecules are completely decoupled. On the other hand, in Ref. [3] the solvent and the ions are modeled as hard spheres with Yukawa attraction between solvent-solvent, solvent-ion, and ion-ion pairs as well as Coulomb interactions between ions. The model was studied by using classic density functional theory (DFT) [6]. Subsequently, the model used in Ref. [4] includes the polar nature of the solvent explicitly, representing its molecules by dipolar hard spheres. In Ref. [5] a lattice model for an electrolyte with nearest-neighbor attraction between all pairs of particles and Coulomb interactions between ions is studied using classic DFT. Although the details of the models used in all these studies differ significantly, all of them agree concerning the trend that electrostatic forces favor first-order wetting transitions. Therefore, the natural question arises whether this observation is accidental or whether there is a deeper reason for it.

Most of the aforementioned studies are based on numerical calculations [3 [5] and only in Refs. [1, 2] analytic expressions for the so-called effective interface potential

\footnotetext{
* ingrid@is.mpg.de

† bier@is.mpg.de
}

[7, 8], which provides all relevant informations about wetting transitions, have been derived and analyzed systematically. However, this analysis is involved because it is based on solutions of non-linear differential equations which show a complex dependence on the relevant parameters.

Here, in order to infer how the order of the wetting transition is affected by the presence of particles with electrostatic interactions, we resort to a suitable model for an electrolyte solution near a charged wall which has been introduced and studied in Ref. [9]. Within this approach we derive an approximate expression for the effective interface potential, the analysis of which provides a transparent understanding of the wetting behavior of electrolyte solutions. In Sec. II we study the case of short-ranged solvent-solvent and short-ranged solventwall interactions. The case of long-ranged solvent-solvent and long-ranged solvent-wall interactions, which has not been considered before, is discussed in Sec. III. We summarize our main results in Sec. [V]

\section{MODEL WITH SHORT-RANGED INTERACTIONS}

We consider a model [9] for an electrolyte solution in three spatial dimensions consisting of solvent molecules, anions (-), and cations $(+)$ close to a charged planar wall. Solvent particles are assumed to have a non-vanishing volume $a^{3}$ whereas the ions are considered to be pointlike particles. The wall under consideration is the $\tilde{x}-\tilde{y}$ plane at $\tilde{z}=0$, i.e., $\tilde{\mathbf{r}}=\left(\tilde{\mathbf{r}}_{\|}=(\tilde{x}, \tilde{y}), \tilde{z}=0\right)$ which can carry a surface charge density $\tilde{\sigma}=\sigma e a^{-2}$, where $e>0$ is the elementary charge. We start from the following variational grand canonical functional, which is a modification 
of the one introduced in Ref. [9]:

$$
\begin{aligned}
\beta \Omega_{0}\left[\phi(\mathbf{r}), \rho_{ \pm}(\mathbf{r})\right] & =\int d^{3} r\left\{\phi(\mathbf{r})\left(\ln (\phi(\mathbf{r}))-\beta \mu_{\phi}\right)\right. \\
& +(1-\phi(\mathbf{r})) \ln (1-\phi(\mathbf{r})) \\
& \left.+\chi(T) \phi(\mathbf{r})(1-\phi(\mathbf{r}))+\frac{\chi(T)}{6}(\nabla \phi(\mathbf{r}))^{2}\right\} \\
& -\beta h_{1} \int d^{2} r_{\|} \phi\left(\mathbf{r}_{\|}, z=0\right) \\
& +\beta \frac{g}{2} \int d^{2} r_{\|} \phi\left(\mathbf{r}_{\| \mid}, z=0\right)^{2} \\
& +\int d^{3} r\left\{\sum _ { i = \pm } \rho _ { i } ( \mathbf { r } ) \left(\ln \rho_{i}(\mathbf{r})\right.\right. \\
& \left.-1-\beta \mu_{i}+V_{i}(\phi(\mathbf{r}))\right) \\
& \left.+\frac{2 \pi l_{B}}{\varepsilon(\phi(\mathbf{r}))}\left(\mathbf{D}\left(\mathbf{r},\left[\rho_{ \pm}\right]\right)\right)^{2}\right\},
\end{aligned}
$$

where $\beta=\left(k_{B} T\right)^{-1}$ is the inverse thermal energy, $\mu_{\phi}$ is the chemical potential of the solvent, $\mu_{ \pm}$are the chemical potentials of the \pm -ions, $\tilde{l}_{B}=l_{B} a=e^{2} \beta /\left(4 \pi \varepsilon_{0}\right)$ is the Bjerrum length in vacuum, and $\mathbf{r}=\tilde{\mathbf{r}} / a$ are dimensionless positions. The actual number density of the solvent is given by $\tilde{\phi}(\mathbf{r})=\phi(\mathbf{r}) a^{-3}$ with $\phi(\mathbf{r}) \in[0,1]$, whereas the number densities of anions and cations are given by $\tilde{\rho}_{ \pm}(\mathbf{r})=\rho_{ \pm}(\mathbf{r}) a^{-3}$. In the following the fluid solvent at position $\mathbf{r}$ with $\phi(\mathbf{r})<1 / 2$ is referred to as a "gas", whereas for $\phi(\mathbf{r})>1 / 2$ it is called a "liquid". The first and the last integral are taken over the half-space $\mathbf{r}=(x, y, z \geq 0)$ whereas the second and the third integral run over the surface $z=0 ; \rho_{ \pm}\left(\ln \rho_{ \pm}-1-\beta \mu_{ \pm}\right)$ is the bulk grand potential density of the \pm -ions in the low number density limit. The Flory-Huggins parameter $\chi(T)>0$ describes the effective interaction between solvent particles [10]. The excess free energy of the solvent $\beta F_{e x}^{\text {sol }}[\phi(\mathbf{r})]=\int d^{3} r\left[\chi(T) \phi(\mathbf{r})(1-\phi(\mathbf{r}))+\frac{\chi(T)}{6}(\nabla \phi(\mathbf{r}))^{2}\right]$ is taken into account using the square-gradient approximation. The ratio $1 / 6$ of the coefficients in the two terms of $\beta F_{e r}^{s o l}[\phi(\mathbf{r})]$ follows from considering nearest neighbors only [1]. Within this model the interaction of the solvent with the wall is captured by the parameters $h_{1}$ and $g$. This implicitly assumes that the fluid-wall interactions are sufficiently short ranged so that their contributions to $\Omega_{0}$ depend only on the solvent density $\phi\left(\mathbf{r}_{\|}, z=0\right)$ in the vicinity of the wall. This parametrization has been used by Nakanishi and Fisher [12] in order to analyze the global surface phase diagram of the LandauGinzburg theory for wetting. $V_{ \pm}(\phi)$ is the solvation free energy per $k_{B} T$ of a \pm -ion in the solvent of number density $\phi$. Whereas more realistic expressions of $V_{ \pm}(\phi)$ are discussed in the literature [9], we use here a simple piece-wise constant expression $V_{ \pm}(\phi<1 / 2)=V_{g}$ and $V_{ \pm}(\phi>1 / 2)=V_{l}$ with $V_{g}-V_{l} \gg 1$. This choice guarantees a vanishingly small ionic strength in the gas $(\phi<1 / 2)$ as compared to the ionic strength in the liquid $(\phi>1 / 2)$. Without restriction of generality we choose
$V_{l}:=0$, which can be achieved by a redefinition of the ionic chemical potentials $\left(\beta \mu_{ \pm}-V_{l} \mapsto \beta \hat{\mu}_{ \pm}\right.$; in the following we drop the hat $\left.{ }^{~}\right)$. The discontinuity of $V_{ \pm}(\phi)$ at $\phi=1 / 2$ is expected to not affect the results significantly because only thermodynamic states of liquid-gas coexistence well below the critical point are considered, for which $\phi=1 / 2$ is deep inside the unstable region of the bulk phase diagram. Note that here no unequal partitioning of ions in a non-uniform solvent occurs due to $V_{+}(\phi)-V_{-}(\phi)=0$, i.e., due to a vanishing difference of solubility contrasts of anions and cations between the two phases in the sense of Ref. [9]. Moreover, no specific adsorption of ions at interfaces is considered here, i.e., there are no surface fields acting on $\rho_{ \pm} \cdot \tilde{\mathbf{D}}=\mathbf{D} e a^{-2}$ is the electric displacement generated by the ions and by the surface charge density as related according to Gauß's law $\nabla \cdot \mathbf{D}\left(\mathbf{r},\left[\rho_{ \pm}\right]\right)=\rho_{+}(\mathbf{r})-\rho_{-}(\mathbf{r})+\sigma \delta(z)$. (Note that Gauß's law is an ingredient of the theory in addition to Eq. (1).) Within the present model, ions interact among each other and with the wall only electrostatically (besides the hard core repulsion of the wall which prevents the ions to penetrate the wall). Here, this is expressed in terms of the energy density of the electric field [5] where $\varepsilon(\phi)$ is the local permittivity of the solvent of density $\phi$ divided by the vacuum permittivity $\varepsilon_{0}$. Various empirical expressions for $\varepsilon(\phi)$ are in use [13]. However, for the sake of simplicity here we adopt a simple piece-wise constant expression $\varepsilon(\phi<1 / 2)=1$ and $\varepsilon(\phi>1 / 2)=\varepsilon_{l}$ with the relative permittivity $\varepsilon_{l}$ of the liquid solvent. For the same reasons as for the case of the piece-wise constant expressions $V_{ \pm}(\phi)$ (see above), the discontinuity of $\varepsilon(\phi)$ at $\phi=1 / 2$ is expected to be irrelevant for the present purposes.

The bulk grand canonical potential density per $k_{B} T$ following from Eq. (1) is given by

$$
\begin{aligned}
\beta \Omega_{b}(\phi, \rho) & =f_{\text {sol }}(\phi)+f_{\text {ion }}^{(+)}(\rho)+f_{\text {ion }}^{(-)}(\rho) \\
& +\rho\left(V_{+}(\phi)+V_{-}(\phi)\right)
\end{aligned}
$$

with $\rho_{+}=\rho_{-}:=\rho$ due to local charge neutrality in the bulk, and with the abbreviations $f_{\text {sol }}(\phi):=\phi(\ln (\phi)-$ $\left.\beta \mu_{\phi}\right)+(1-\phi) \ln (1-\phi)+\chi(T) \phi(1-\phi)$ and $f_{\text {ion }}^{( \pm)}\left(\rho_{ \pm}\right):=$ $\rho_{ \pm}\left(\ln \rho_{ \pm}-1-\beta \mu_{ \pm}\right)$. As a consequence of local charge neutrality $\Omega_{b}$ depends on $\mu_{+}$and $\mu_{-}$only via the combination $\mu_{+}+\mu_{-} \equiv \mu_{I}$. Accordingly, the ionic chemical potentials $\mu_{ \pm}$are of no individual importance but only their sum is of physical relevance. In the bulk $D=0$ due to local charge neutrality so that the last term in Eq. (1) does not contribute to Eq. (2). Equilibrium bulk states $(\phi, \rho)$ minimize $\beta \Omega_{b}\left(\phi, \rho ; \mu_{\phi}, \mu_{I}, T\right)$, i.e., they fulfill the Euler-Lagrange equations

$$
\frac{\partial \Omega_{b}}{\partial \phi}=0
$$

and

$$
\frac{\partial \Omega_{b}}{\partial \rho}=0 .
$$


Equations (3) and (4) render two solutions, i.e., minima: $\quad\left[\phi_{l}\left(\mu_{\phi}, \mu_{I}, T\right), \rho_{l}\left(\mu_{\phi}, \mu_{I}, T\right) \equiv I\right] \quad$ and $\left[\phi_{g}\left(\mu_{\phi}, \mu_{I}, T\right), \rho_{g}\left(\mu_{\phi}, \mu_{I}, T\right)\right]$ Coexistence between these two minima occurs if upon inserting these two solutions into $\Omega_{b}$ the minima are equally deep:

$$
\begin{array}{r}
\Omega_{b}\left(\phi=\phi_{l}\left(\mu_{\phi}, \mu_{I}, T\right), \rho=\rho_{l}\left(\mu_{\phi}, \mu_{I}, T\right) ; \mu_{\phi}, \mu_{I}, T\right) \\
=\Omega_{b}\left(\phi=\phi_{g}\left(\mu_{\phi}, \mu_{I}, T\right), \rho=\rho_{g}\left(\mu_{\phi}, \mu_{I}, I\right) ; \mu_{\phi}, \mu_{I}, T\right) .
\end{array}
$$

This renders a relation $\mu_{\phi}=\mu_{\phi}^{c o}\left(\mu_{I}, T\right)$ which describes a two-dimensional manifold in the three-dimensional parameter space $\left(\mu_{\phi}, \mu_{I}, T\right)$ where gas-liquid coexistence occurs. Inserting this relations into the solutions ren$\operatorname{ders}\left[\phi_{l}^{c o}\left(\mu_{I}, T\right), \rho_{l}^{c o}\left(\mu_{I}, T\right)\right]$ and $\left[\phi_{g}^{c o}\left(\mu_{I}, T\right), \rho_{g}^{c o}\left(\mu_{I}, T\right)\right]$ with $\phi_{l, g}^{c o}\left(\mu_{I}, T\right)=\phi_{l, g}\left(\mu_{\phi}=\mu_{\phi}^{c o}\left(\mu_{I}, T\right), \mu_{I}, T\right)$ and $\rho_{l, g}^{c o}\left(\mu_{I}, T\right)=\rho_{l, g}\left(\mu_{\phi}=\mu_{\phi}^{c o}\left(\mu_{I}, T\right), \mu_{I}, T\right)$ where $\rho_{l}^{c o}=$ $I$. In order to avoid a clumsy notation, in the following we drop the superscript co in $\phi_{l}^{c o}, \phi_{g}^{c o}$, and $\rho_{g}^{c o}$ so that, if not stated otherwise, $\phi_{l}, \phi_{g}, I$, and $\rho_{g}$ correspond to the coexisting densities.

Equation (4) can be used to express the bulk ionic strength as

$$
\rho=\exp \left(\frac{1}{2}\left(\beta \mu_{I}-V_{+}(\phi)-V_{-}(\phi)\right)\right) .
$$

Due to our choice $V_{ \pm}(\phi<1 / 2)=V_{g}$ and $V_{ \pm}(\phi>1 / 2)=$ $V_{l}=0$ we obtain for the ionic strength in the gas $(\phi=$ $\left.\phi_{g}<1 / 2\right) \rho=\rho_{g}=\exp \left(\beta \mu_{I} / 2-V_{g}\right)$ and in the liquid $\left(\phi=\phi_{l}>1 / 2\right) \rho=\rho_{l}=\exp \left(\beta \mu_{I} / 2\right)$ (see Eq. (6) ). In the following it is assumed that $V_{g} \gg 1$ such that we can set $\rho_{g}=0$, and $I=\rho_{l}=\exp \left(\beta \mu_{I} / 2\right)$. Accordingly, by using $\mu_{I}=2 k_{B} T \ln I$ the densities discussed above can be expressed as functions of $I$ and $T$. Note that Eq. (6) is independent of the Flory-Huggins parameter $\chi(T)$. For our choice of the ion potential $V_{ \pm}(\phi)$ (such that $\left.V_{ \pm}^{\prime}(\phi \neq 1 / 2)=V_{ \pm}^{\prime \prime}(\phi \neq 1 / 2)=0\right)$ the binodal $T_{b i}(\phi)$ is determined via the implicit relation

$$
\chi(T)=\frac{\ln (\phi)-\ln (1-\phi)}{2 \phi-1},
$$

where the temperature dependence of $\chi(T)$ is often taken to be $\chi(T) \cong \chi_{S}+\chi_{H} / T$, where $\chi_{S}$ and $\chi_{H} / T$ are referred to as the entropic and the enthalpic part of $\chi(T)$, respectively [10]. From Eq. (7) one infers the critical point to be located at $\left(\phi_{c}=1 / 2, \chi\left(T_{c}\right)=2\right)$. Note that within our approximation the binodal (and hence the critical point) is independent of the ionic strength $I$.

In the presence of walls, $\phi$ and $\rho_{ \pm}$vary spatially in normal direction $z$. Their equilibrium profiles minimize the full functional $\Omega_{0}\left[\phi(\mathbf{r}), \rho_{ \pm}(\mathbf{r})\right]$ in Eq. (1) and thus render the equilibrium state. This procedure can be performed numerically. However, for the present purpose, we seek analytic expressions. In order to achieve this goal we perform a Taylor expansion of the local part in Eq. (1) around the $s$ harp- $k$ ink reference density profiles [8]

$$
\bar{\phi}(z)=\phi_{s k}(z)= \begin{cases}\phi_{l}, & 0 \leq z \leq \ell \\ \phi_{g}, & z>\ell\end{cases}
$$

and

$$
\bar{\rho}_{ \pm}(z)=\rho_{s k, \pm}(z)= \begin{cases}I, & 0 \leq z \leq \ell \\ 0, & z>\ell\end{cases}
$$

where $\ell$ is the position of the discontinuity of the sharpkink profile $\phi_{s k}(z)$, and $\phi_{l}$ and $\phi_{g}$ are, respectively, the equilibrium bulk densities of the solvent in the liquid and gas phase for a bulk ionic strength $I$ in the liquid phase. This Taylor expansion renders an approximate variational functional $\hat{\Omega}_{0}$ which up to quadratic order is given by

$$
\begin{aligned}
\frac{\beta \hat{\Omega}_{0}\left[\phi(z), \rho_{ \pm}(z)\right]}{A} & =\ell f_{\text {sol }}\left(\phi_{l}\right)+(L-\ell) f_{\text {sol }}\left(\phi_{g}\right) \\
& +\int_{0}^{L} d z\left\{f_{\text {sol }}^{\prime}(\bar{\phi}(z))(\phi(z)-\bar{\phi}(z))\right. \\
& +\frac{1}{2} f_{\text {sol }}^{\prime \prime}(\bar{\phi}(z))(\phi(z)-\bar{\phi}(z))^{2} \\
& \left.+\frac{\chi(T)}{6}\left(\frac{d \phi(z)}{d z}\right)^{2}\right\} \\
& -\beta h_{1} \phi(0)+\beta \frac{g}{2}(\phi(0))^{2} \\
& +\int_{0}^{\ell} d z\left\{\sum _ { i = \pm } \left[f_{\text {ion }}^{(i)}(I)\right.\right. \\
& +f_{\text {ion }}^{(i)^{\prime}}(I)\left(\rho_{i}(z)-I\right) \\
& \left.+\frac{1}{2} f_{\text {ion }}^{(i)}{ }^{\prime \prime}(I)\left(\rho_{i}(z)-I\right)^{2}\right] \\
& \left.+\frac{2 \pi l_{B}}{\varepsilon_{l}}\left(D\left(z,\left[\rho_{ \pm}\right]\right)\right)^{2}\right\}
\end{aligned}
$$

where $\tilde{A}=A a^{2}$ is the wall area and $\tilde{V}=A L a^{3}$ is the volume of the system. In order to obtain Eq. (10) it has been used that $\rho_{ \pm}(z>\ell)=0, V_{ \pm}(\phi(z \leq \ell))=V_{l}=0$ because $\phi(z \leq \ell)>1 / 2$, and $\varepsilon(\phi \leq \ell)=\varepsilon_{l}$. Therefore Eq. (10) does not apply very close to the critical point where the actual spatial variation of $V_{ \pm}(\phi(z))$ and $\varepsilon(\phi(z))$ matters. Moreover, $D(z>\ell)=0$ because the gas phase contains no ions and $D(z \rightarrow \infty) \rightarrow 0$ due to the constraint of global charge neutrality.

The Euler-Lagrange equation for $\phi(z)$, which follows from Eq. (10) for fixed $\ell$, is given by

$$
\frac{\chi(T)}{3} \frac{d^{2} \phi(z)}{d z^{2}}=f_{\text {sol }}^{\prime}(\bar{\phi}(z))+f_{\text {sol }}^{\prime \prime}(\bar{\phi}(z))(\phi(z)-\bar{\phi}(z))
$$


with the boundary conditions

$\left.\frac{\chi(T)}{3} \frac{d \phi(z)}{d z}\right|_{z=0}=-\beta h_{1}+\beta g \phi(0)$ and $\left.\frac{d \phi(z)}{d z}\right|_{z=L}=0$.

Similarly, the Euler-Lagrange equations for $\rho_{ \pm}(z), 0 \leq$ $z \leq \ell$, read (using Eq. (14) and $\frac{d}{d z} \frac{\delta D(z)}{\delta \rho_{i}\left(z^{\prime}\right)}=\frac{\delta}{\delta \rho_{i}\left(z^{\prime}\right)} \frac{d D(z)}{d z}=$ $q_{i} \delta\left(z-z^{\prime}\right)$ due to Gauß's law $)$

$$
f_{\text {ion }}^{(i)^{\prime}}(I)+f_{\text {ion }}^{(i)^{\prime \prime}}(I)\left(\rho_{i}(z)-I\right)=-q_{i} \Psi(z),
$$

where $e q_{ \pm}$is the ion charge with $q_{ \pm}= \pm 1$ and $\tilde{\Psi}(z)=$ $\Psi(z) /(\beta e)$ is the electrostatic potential such that

$$
D(z)=-\frac{\varepsilon_{l}}{4 \pi l_{B}} \Psi^{\prime}(z) \quad \text { for } 0 \leq z \leq \ell .
$$

The variation leading to Eq. (13) generates also boundary terms $\Psi(z) \delta D\left(z,\left[\rho_{ \pm}\right]\right) / \delta \rho_{i}\left(z^{\prime}\right)$ at $z=0$ and $z=\ell$ which, however, vanish because of the boundary conditions $D(z=0)=\sigma$ and $D(z=\ell)=0$. The latter holds due to $D \equiv 0$ in the gas and the continuity of $D(z)$ at $z=\ell$ in the absence of a surface charge at $z=\ell$. Due to Eq. (41), in Eq. (13) one has $f_{\text {ion }}^{(i)^{\prime}}=0$. For the particular choice of $\bar{\phi}(z)$ in Eq. (8) one has $f_{\text {sol }}^{\prime}(\bar{\phi}(z<\ell))=$ $f_{\text {sol }}^{\prime}\left(\phi_{l}\right)=0$ and $f_{\text {sol }}^{\prime}(\bar{\phi}(z>\ell))=f_{\text {sol }}^{\prime}\left(\phi_{g}\right)=0$ due to Eq. (3) and the Euler-Lagrange equation (11) can be written as

$$
\frac{d^{2}}{d z^{2}} \Delta \phi(z)= \begin{cases}\frac{1}{\xi_{l}^{2}} \Delta \phi(z), & 0 \leq z<\ell \\ \frac{1}{\xi_{g}^{2}} \Delta \phi(z), & z>\ell\end{cases}
$$

with

$$
\frac{1}{\xi_{g, l}^{2}}=\frac{3}{\chi(T)}\left(\frac{1}{\phi_{g, l}}+\frac{1}{1-\phi_{g, l}}-2 \chi(T)\right),
$$

where $\xi_{g, l}$ can be identified with the bulk correlation length of the solvent in the gas and in the liquid phase, respectively (see Appendix $\mathrm{B}$ ), and where $\Delta \phi(z)=\phi(z)-$ $\bar{\phi}(z)=\phi(z)-\phi_{l}$ for $0 \leq z<\ell$ and $\Delta \phi(z)=\phi(z)-\phi_{g}$ for $z>\ell$. In addition to the boundary conditions in Eq. (12), Eq. (11) requires the density profile $\phi(z)$ and its first derivative $\frac{d \phi(z)}{d z}$ to be continuous at $z=\ell$, i.e.,

$$
\begin{aligned}
\phi_{l}+\Delta \phi\left(\ell^{-}\right) & =\phi_{g}+\Delta \phi\left(\ell^{+}\right) \\
\left.\frac{d}{d z} \Delta \phi(z)\right|_{z=\ell^{-}} & =\left.\frac{d}{d z} \Delta \phi(z)\right|_{z=\ell^{+}}
\end{aligned}
$$

because the right-hand side of Eq. (11) is discontinuous; otherwise the left-hand side of Eq. (11) would be more singular at $z=\ell$ than the right-hand side. Similarly, for $\Delta \rho_{ \pm}(z)$ one obtains (see Eqs. (4) and (13))

$$
\Delta \rho_{ \pm}(z)=-q_{ \pm} \Psi(z) I
$$

where $\Delta \rho_{ \pm}(z)=\rho_{ \pm}(z)-\bar{\rho}_{ \pm}(z)=\rho_{ \pm}(z)-I$ for $0 \leq z<\ell$ and zero otherwise (see Eq. (9) and $\rho_{ \pm}(z>\ell)=0$ ). The dependences of Eqs. (11) and (18) on $\mu_{\phi}$ and $\mu_{I}$ enter via the equilibrium values of $I, \phi_{g}$, and $\phi_{l}$ (Eqs. (2)-(5)).

The Poisson equation, which relates the dimensionless electrostatic potential $\Psi=\beta e \tilde{\Psi}$ to the dimensionless number densities $\rho_{ \pm}$of the ions, can be written as (see Eqs. (14) and (18) and Gauß's law)

$$
\begin{aligned}
\Psi^{\prime \prime}(z)=-\frac{4 \pi \lambda_{B}}{\varepsilon_{l}} \frac{d D}{d z} & =-\frac{4 \pi l_{B}}{\varepsilon_{l}} \sum_{i= \pm} q_{i} \rho_{i}(z) \\
& =-\frac{4 \pi l_{B}}{\varepsilon_{l}} \sum_{i= \pm} q_{i}\left(\Delta \rho_{i}+I\right) \\
& =-\frac{4 \pi l_{B}}{\varepsilon_{l}} \sum_{i= \pm} q_{i} I\left(1-q_{i} \Psi(z)\right) \\
& =\frac{8 \pi I l_{B}}{\varepsilon_{l}} \Psi(z) \\
& =\kappa^{2} \Psi(z)
\end{aligned}
$$

which is the linearized Poisson-Boltzmann equation with

$$
\kappa=\sqrt{8 \pi I l_{B} / \varepsilon_{l}}
$$

as the inverse Debye length. This equation must be solved subject to the boundary conditions

$$
\Psi^{\prime}(z=0)=-\frac{4 \pi l_{B} \sigma}{\varepsilon_{l}} \text { and } \Psi^{\prime}(z=\ell)=0
$$

which corresponds to $D(z=0)=\sigma$ and $D(z=\ell)=0$; the latter follows from the overall charge neutrality (see Eqs. (13) and (14) in Ref. [5]) and the assumption that there are no ions in the vapor phase. Equations (15) and (19) can be solved analytically, yielding the constrained equilibrium profiles

$$
\Delta \phi^{(\ell)}(z)= \begin{cases}\hat{A}_{l} \exp \left(z / \xi_{l}\right)+\hat{B}_{l} \exp \left(-z / \xi_{l}\right), & 0 \leq z \leq \ell \\ \hat{B}_{g} \exp \left(-z / \xi_{g}\right), & z>\ell\end{cases}
$$

where (see Eqs. (17) and (12))

$$
\begin{aligned}
\hat{A}_{l} & =\frac{1}{N}\left[\left(\beta g+\frac{\chi(T)}{3 \xi_{l}}\right)\left(\phi_{g}-\phi_{l}\right)\right. \\
& \left.\left.+\beta\left(h_{1}-g \phi_{l}\right)\left(\frac{\xi_{g}}{\xi_{l}}-1\right) \exp \left(-\ell / \xi_{l}\right)\right)\right], \\
\hat{B}_{l} & =\frac{1}{N}\left[\left(\frac{\chi(T)}{3 \xi_{l}}-\beta g\right)\left(\phi_{g}-\phi_{l}\right)\right. \\
& \left.\left.+\beta\left(h_{1}-g \phi_{l}\right)\left(1+\frac{\xi_{g}}{\xi_{l}}\right) \exp \left(\ell / \xi_{l}\right)\right)\right], \\
\hat{B}_{g} & =\frac{\xi_{g}}{\xi_{l}}\left(B_{l} \exp \left(\frac{\ell}{\xi_{g}}-\frac{\ell}{\xi_{l}}\right)-\hat{A}_{l} \exp \left(\frac{\ell}{\xi_{g}}+\frac{\ell}{\xi_{l}}\right)\right), \\
\hat{N} & =\left(\beta g+\frac{\chi(T)}{3 \xi_{l}}\right)\left(\frac{\xi_{g}}{\xi_{l}}+1\right) \exp \left(\ell / \xi_{l}\right) \\
& -\left(\beta g-\frac{\chi(T)}{3 \xi_{l}}\right)\left(1-\frac{\xi_{g}}{\xi_{l}}\right) \exp \left(-\ell / \xi_{l}\right),
\end{aligned}
$$


and (see Eq. (18) $) \Delta \rho_{ \pm}^{(\ell)}(z)=-q_{ \pm} I \Psi^{(\ell)}(z)$ with

$$
\Psi^{(\ell)}(z)=A_{I} \exp (\kappa z)+B_{I} \exp (-\kappa z),
$$

where (see Eq. (21))

$$
\begin{aligned}
A_{I} & =\frac{4 \pi l_{B} \sigma}{\varepsilon_{l} \kappa} \frac{1}{\exp (2 \kappa \ell)-1}, \\
B_{I} & =A_{I} \exp (2 \kappa \ell) .
\end{aligned}
$$

Note that because $\rho_{ \pm}(z) \geq 0,\left|\Delta \rho_{ \pm}(z)\right|$ has an upper limit given by (see Eq. (18))

$$
\left|\Delta \rho_{ \pm}(z)\right| \leq I \text {, i.e., }|\Psi(z)| \leq 1,
$$

Since $\Psi(z)$ is monotonic (see Eqs. (24) and (25)) one requires

$$
\begin{aligned}
|\Psi(0)| & \leq 1 \\
\Rightarrow \frac{4 \pi l_{B}|\sigma|}{\varepsilon_{l} \kappa} \operatorname{coth}(\kappa \ell) & \leq 1,
\end{aligned}
$$

which implies that there is an upper limit for the absolute value $|\sigma|$ surface charge density:

$$
|\sigma| \leq \frac{\varepsilon_{l} \kappa}{4 \pi l_{B} q_{ \pm}} \tanh (\kappa \ell)=: \sigma_{s a t}(\kappa \ell)
$$

In case the real surface charge $|\sigma|$ is larger than the saturation value $\sigma_{\text {sat }}(\kappa \ell)$ the latter has to be used instead in order to ensure $\rho_{ \pm}(z) \geq 0$. This is the analogue of the well-known charge renormalization in the linearized Poisson-Boltzmann theory of semi-infinite electrolyte solutions [14].

Within the present model, at two-phase coexistence $\phi_{g}=1-\phi_{l}$ so that $\xi_{g}=\xi_{l}=\xi$ and that Eq. (23) reduces to

$$
\begin{aligned}
A_{l} & =\frac{1}{N}\left(\beta g+\frac{\chi(T)}{3 \xi}\right)\left(\phi_{g}-\phi_{l}\right) \\
B_{l} & =\frac{1}{N}\left[\left(\frac{\chi(T)}{3 \xi}-\beta g\right)\left(\phi_{g}-\phi_{l}\right)\right. \\
& \left.\left.+2 \beta\left(h_{1}-g \phi_{l}\right) \exp (\ell / \xi)\right)\right] \\
B_{g} & =\left(B_{l}-A_{l} \exp (2 \ell / \xi)\right) \\
N & =2\left(\beta g+\frac{\chi(T)}{3 \xi}\right) \exp (\ell / \xi)
\end{aligned}
$$

The solvent density profiles at two-phase coexistence obtained by expanding $\Omega_{0}$ up to quadratic order around the sharp-kink profiles (see Eqs. (22) and (29) - are similar but not identical to the ones obtained by using the so-called double parabola approximation (DPA) (see Appendix A). A difference between the two approaches arises because the boundary conditions and the definition of the thickness of the liquid film differ in both approaches. Within the present approach the thickness $\ell_{\text {film }}$ of the liquid film is defined as the position $z>0$ in which the magnitude of the derivative $\left|\phi^{\prime}(z)\right|$ of the solvent profile is maximal. This definition of the film thickness $\ell_{\text {film }}$ is convenient within the present approach because Eq. (22) shows that it coincides with the position $\ell$ of the discontinuity of the sharp-kink profile $\phi_{s k}(z)$, i.e., $\ell_{\text {film }}=\ell$. Alternative definitions of the film thickness are possible but lead to more complicated expressions of the effective interface potential introduced below. Moreover, the present solvent profile and its derivative are continuous everywhere (see Eq. (17)), whereas within the DPA the solvent profile is continuous everywhere but its derivative is discontinuous at the position $z=\ell_{\mathrm{DP}}$ where $\phi_{\mathrm{DP}}\left(\ell_{\mathrm{DP}}\right)=\frac{1}{2}\left(\phi_{g}+\phi_{l}\right)$ (see Eq. (A7). This equation defines the liquid film thickness $\ell_{\text {DPA }}$ within the DPA. However, the discontinuity $\phi_{\mathrm{DPA}}^{\prime}\left(\ell_{\mathrm{DPA}}^{+}\right)-\phi_{\mathrm{DPA}}^{\prime}\left(\ell_{\mathrm{DPA}}^{-}\right)=\mathcal{O}\left(\exp \left(-\ell_{\mathrm{DP}} / \xi\right)\right)$ is exponentially small for large film thicknesses $\left(\ell_{\mathrm{DPA}} \gg \xi\right)$ (see Eqs. (A7) and (A8). Moreover, in Appendix A it is shown that the relative difference between the coefficients of the profiles in Eq. (22) and those in Eq. (A7) is also exponentially small for $\ell=\ell_{\mathrm{DPA}} \gg \xi$ (see Eq. (A9)).

At the functional minimum the integrations in Eq. (10) can be performed analytically with the first integrand (see Eqs. (15) and (16)

$$
\begin{aligned}
\frac{\chi(T)}{6}\left(\frac{d}{d z}\right. & \Delta \phi(z))^{2}+\frac{1}{2}(\Delta \phi(z))^{2}\left(\frac{1}{\phi_{g, l}}+\frac{1}{1-\phi_{g, l}}-2 \chi(T)\right) \\
& =\frac{\chi(T)}{6}\left(\frac{(\Delta \phi(z))^{2}}{\xi_{g, l}^{2}}+\left(\frac{d}{d z} \Delta \phi(z)\right)^{2}\right) \\
& =\frac{\chi(T)}{6}\left(\frac{(\Delta \phi(z))^{2}}{\xi_{g, l}^{2}}+\frac{d}{d z}\left(\Delta \phi(z) \frac{d}{d z} \Delta \phi(z)\right)\right. \\
& \left.-\Delta \phi(z) \frac{d^{2}}{d z^{2}}(\Delta \phi(z))\right) \\
& =\frac{\chi(T)}{6} \frac{d}{d z}\left(\Delta \phi(z) \frac{d}{d z} \Delta \phi(z)\right)
\end{aligned}
$$

and with the second the integrand (see Eqs. (14), (18), and (19) and $f_{\text {ion }}^{(i)^{\prime}}(I)=0$ )

$$
\begin{aligned}
\frac{1}{2 I} \sum_{i= \pm}\left(\Delta \rho_{i}(z)\right)^{2} & +\frac{2 \pi l_{B}}{\varepsilon_{l}}\left(D\left(z,\left[\Delta \rho_{ \pm}\right]\right)\right)^{2} \\
& =\frac{I}{\kappa^{2}}\left(\kappa^{2}(\Psi(z))^{2}+\left(\Psi^{\prime}(z)\right)^{2}\right) \\
& =\frac{I}{\kappa^{2}}\left(\Psi(z) \Psi^{\prime \prime}(z)+\left(\Psi^{\prime}(z)\right)^{2}\right) \\
& =\frac{I}{\kappa^{2}}\left(\Psi(z) \Psi^{\prime}(z)\right)^{\prime} .
\end{aligned}
$$

By exploiting the boundary conditions in Eqs. (12), (17), and (21) one obtains for the surface contribution to the 
constrained grand potential

$$
\begin{aligned}
\beta \Omega_{s}(\ell):= & \frac{\beta \hat{\Omega}_{0}\left[\phi^{(\ell)}(z), \rho_{ \pm}^{(\ell)}(z)\right]-V \beta \Omega_{b}\left(\phi_{g}, \rho_{g}=0\right)}{A} \\
= & \beta \ell\left[\Omega_{b}\left(\phi_{l}, I\right)-\Omega_{b}\left(\phi_{g}, 0\right)\right] \\
& +\frac{\chi(T)}{6}\left(\Delta \phi^{(\ell)^{\prime}}(\ell)\left(\phi_{g}-\phi_{l}\right)-\Delta \phi^{(\ell)^{\prime}}(0) \Delta \phi^{(\ell)}(0)\right) \\
& -\beta h_{1}\left(\phi_{l}+\Delta \phi^{(\ell)}(0)\right)+\frac{\beta g}{2}\left(\phi_{l}+\Delta \phi^{(\ell)}(0)\right)^{2} \\
& +\frac{1}{2} \sigma \Psi^{(\ell)}(0)
\end{aligned}
$$

where $\Delta \phi^{(\ell)^{\prime}}(\ell)=\left.\left(\frac{d}{d z} \Delta \phi^{(\ell)}(z)\right)\right|_{z=\ell}$. Finally, inserting the solutions given by Eqs. (22)-(25) into Eq. (32) and using Eqs. (12) and (21) leads to

$$
\begin{aligned}
\beta \Omega_{s}(\ell) & =\beta \ell\left[\Omega_{b}\left(\phi_{l}, I\right)-\Omega_{b}\left(\phi_{g}, 0\right)\right] \\
& +\frac{\chi(T)}{6}\left(\phi_{g}-\phi_{l}\right)\left[\frac{\hat{A}_{l}}{\xi_{l}} \exp \left(\ell / \xi_{l}\right)\right. \\
& \left.-\frac{\hat{B}_{l}}{\xi_{l}} \exp \left(-\ell / \xi_{l}\right)\right] \\
& +\frac{\beta\left(g \phi_{l}-h_{1}\right)}{2}\left(\hat{A}_{l}+\hat{B}_{l}\right)-\beta h_{1} \phi_{l}+\frac{\beta g}{2} \phi_{l}^{2} \\
& +\frac{2 \pi l_{B} \sigma^{2}}{\varepsilon_{l} \kappa} \operatorname{coth}(\kappa \ell) .
\end{aligned}
$$

The first term is the difference between the grand canonical potentials per volume of the (potentially metastable) liquid and the gas bulk phase, respectively, multiplied by the film thickness $\ell$. This contribution linear in $\ell$ vanishes at two-phase coexistence due to Eq. (5). The other terms provide the free energy associated with the emergence of the liquid-gas and the liquid-wall interfaces as well as their effective interaction for $\ell<\infty$. These expressions are valid also off two-phase coexistence.

At two-phase coexistence and in the limit $\ell \gg 1 / \kappa$ the surface contribution (Eq. (33)) can be written as

$$
\begin{aligned}
\beta \Omega_{s}(\ell) & \simeq \frac{\chi(T)}{12} \frac{\left(\phi_{g}-\phi_{l}\right)^{2}}{\xi}-\frac{\beta^{2}\left(h_{1}-g \phi_{l}\right)^{2}}{2\left(\beta g+\frac{\chi(T)}{3 \xi}\right)} \\
& -\beta h_{1} \phi_{l}+\frac{\beta g}{2} \phi_{l} \\
& +\left(\phi_{l}-\phi_{g}\right) \frac{\chi(T)}{3 \xi} \frac{\beta\left(h_{1}-g \phi_{l}\right)}{\beta g+\frac{\chi(T)}{3 \xi}} \exp (-\ell / \xi) \\
& -\left(\phi_{l}-\phi_{g}\right)^{2} \frac{\chi(T)}{12 \xi} \frac{\frac{\chi(T)}{3 \xi}-\beta g}{\beta g+\frac{\chi(T)}{3 \xi}} \exp (-2 \ell / \xi) \\
& +\frac{2 \pi l_{B} \sigma^{2}}{\varepsilon_{l} \kappa}(1+2 \exp (-2 \kappa \ell)),
\end{aligned}
$$

where only the last term in Eq. (33) has been expanded for $\kappa \ell \gg 1$. Note that within the present theory the ions enter into $\Omega_{s}(\ell)$ only via the last term. Therefore, if the surface charge $\sigma$ is zero, the ions do not modify the wetting behavior of the solvent. This is due to the fact that $\sigma=0$ implies that there are no surface fields acting on $\rho_{ \pm}$. The first term in Eq. (34) is the liquid-gas surface tension $\gamma_{l, g}=\frac{\chi(T)}{12} \frac{\left(\phi_{g}-\phi_{l}\right)^{2}}{\xi}$, within the present approach. As expected, within mean field theory (MFT) $\phi_{l}-\phi_{g}$ vanishes $\propto\left|\chi-\chi_{c}\right|^{\beta}$ with $\beta=1 / 2$ upon approaching the critical point $\left(\phi_{c}=\frac{1}{2}, \chi_{c}=2\right)$ (see Eq. (77) ) and from Eq. (16) one has $\xi \propto\left|\chi-\chi_{c}\right|^{-\nu}$ with $\nu=1 / 2$, so that $\gamma_{l, g} \propto\left|\chi-\chi_{c}\right|^{\mu}$ with $2 \beta+\nu=\mu=3 / 2$; in general $\mu=(d-1) \nu$ where $d$ is the spatial dimension with $d=4$ corresponding to MFT [7]. The wall-liquid surface tension is $\gamma_{w, l}=-\frac{\beta^{2}\left(h_{1}-g \phi_{l}\right)^{2}}{2\left(\beta g+\frac{\chi(T)}{3 \xi}\right)}-\beta h_{1} \phi_{l}+\frac{\beta g}{2} \phi_{l}+\frac{2 \pi l_{B} \sigma^{2}}{\varepsilon_{l} \kappa}$. These two contributions are independent of the film thickness $\ell$. The remaining terms carry the dependence on $\ell$, generated by the effective interaction between the emerging liquid-vapor and wall-liquid interfaces.

Accordingly, the effective interface potential $\omega(\ell)=$ $\Omega_{s}(\ell)-\Omega_{s}(\infty)$ at two-phase coexistence is given by

$$
\begin{aligned}
& \beta \omega(\ell \gg 1 / \kappa) \simeq\left(\phi_{l}-\phi_{g}\right) \frac{\chi(T)}{3 \xi} \frac{\beta\left(h_{1}-g \phi_{l}\right)}{\beta g+\frac{\chi(T)}{3 \xi}} \exp (-\ell / \xi) \\
& -\left(\phi_{l}-\phi_{g}\right)^{2} \frac{\chi(T)}{12 \xi} \frac{\frac{\chi(T)}{3 \xi}-\beta g}{\beta g+\frac{\chi(T)}{3 \xi}} \exp (-2 \ell / \xi) \\
& +\frac{4 \pi l_{B} \sigma^{2}}{\varepsilon_{l} \kappa} \exp (-2 \kappa \ell) \text {. }
\end{aligned}
$$

This effective potential captures the dependence of the grand canonical potential on the film thickness and determines whether or not the wall-gas interface is wetted by the liquid. Moreover, the order of the wetting transition can be inferred from its functional form [8].

The property $\xi_{l}=\xi_{g}$ at coexistence is a special feature of the present model. In general $\xi_{l} \neq \xi_{g}$ so that in this case an expansion of the effective interface potential $\omega(\ell)$ similar to Eq. 351 contains products of powers of $\exp \left(-\ell / \xi_{l}\right)$ and $\exp \left(-\ell / \xi_{g}\right)$ (see Eq. (33)).

\section{A. Pure solvent}

We first consider the case of a pure solvent (i.e., $I=$ 0 ) near a neutral wall (i.e., $\sigma=0$ ) and at gas-liquid coexistence. For such a system the effective interface potential in Eq. (35) reduces to

$$
\beta \omega(\ell)=a_{0}(T) \exp (-\ell / \xi)+b_{0}(T) \exp (-2 \ell / \xi)
$$

with

$$
a_{0}(T)=\left(\phi_{l}-\phi_{g}\right) \frac{\chi(T)}{3 \xi} \frac{\beta\left(h_{1}-g \phi_{l}\right)}{\beta g+\frac{\chi(T)_{2}}{3 \xi}}
$$

and

$$
b_{0}(T)=-\left(\phi_{l}-\phi_{g}\right)^{2} \frac{\chi(T)}{12 \xi} \frac{\frac{\chi(T)}{3 \xi}-\beta g}{\beta g+\frac{\chi(T)}{3 \xi}} .
$$


For second-order wetting to occur at $T=T_{\mathrm{w}}$, the coefficient $a_{0}(T)$ must be negative for $T<T_{\mathrm{w}}$, vanish at $T=T_{\mathrm{w}}$, and be positive for $T>T_{\mathrm{w}}$. As $\phi_{l}>\phi_{g}$, and because $\phi_{l}$ can vary only between its value at the triple point $\phi_{l}\left(T_{t}\right)$ and the critical density $\phi_{c}=\phi_{l}\left(T_{c}\right), a_{0}(T)$ fulfills the above mentioned conditions if

$$
\phi_{c}<\frac{h_{1}}{g}<\phi_{l}\left(T_{t}\right) .
$$

Here and the following we consider $h_{1}>0$ and $g>0$. The order of the transition is determined by the higherorder coefficients in the expansion of $\omega(\ell)$ [8]. If $b_{0}\left(T_{\mathrm{w}}\right)<$ 0 , the transition will be first order while second-order wetting can occur if $b_{0}\left(T_{\mathrm{w}}\right)>0$. Only in the latter case $a_{0}\left(T_{\mathrm{w}}\right)=0$ determines the wetting transition temperature, so that

$$
\phi_{l}\left(T_{\mathrm{w}}\right)=\frac{h_{1}}{g} .
$$

Within the present approach, the wetting transition can be second order if

$$
\beta g>\frac{\chi\left(T_{\mathrm{w}}\right)}{3 \xi}
$$

and first order if the inequality is reversed. The separatrix between first- and second-order wetting (i.e., the loci of tricritical wetting [12]) is given by

$$
\beta g=\frac{\chi\left(T_{\mathrm{w}}\right)}{3 \xi\left(T_{\mathrm{w}}\right)}
$$

where $\chi\left(T_{\mathrm{w}}\right)$ follows from Eqs. (7) and (40):

$$
\chi\left(T_{\mathrm{w}}\right)=\frac{\ln \left(h_{1} / g\right)-\ln \left(1-h_{1} / g\right)}{2 h_{1} / g-1} .
$$

\section{B. Electrolyte solution}

In the case of an electrolyte solution close to a charged wall the effective interface potential given by Eq. (35) has the generic form studied by Aukrust and Hauge [15] for a model in which both the wall-fluid and the fluid-fluid interaction potentials decay exponentially but on distinct scales. If we proceed analogously to extract the information about the wetting behavior as before, we realize that the electrostatic term $a_{I}(T) \exp (-2 \kappa \ell)$ with

$$
a_{I}(T)=\frac{4 \pi l_{B} \sigma^{2}}{\varepsilon_{l} \kappa}
$$

has a coefficient which is always positive. (Equation 35) shows that the coefficients $a_{0}(T)$ (Eq. (37)) and $b_{0}(T)$ (Eq. (38) ) do not change upon adding ions.) Accordingly, the wetting behavior will depend on the competition between the Debye length $1 / \kappa$ and the correlation length $\xi$ : (i) $1 / \kappa<\xi$ : In this case the electrostatic term decays faster than the remaining two terms in Eq. (35). Therefore one obtains the same wetting behavior as for the pure solvent (see Subsec. 【A).

(ii) $\xi<1 / \kappa<2 \xi$ : In this case the electrostatic term is the dominant subleading contribution in the expansion. Moreover, because $a_{I}(T)>0$ for all temperatures, the transition can be second order if $a_{0}(T)$ satisfies the conditions given by Eq. (39).

(iii) $1 / \kappa>2 \xi:$ In this case, the electrostatic term is the leading contribution. As a result, if in the pure solvent the wetting transition is second order, due to adding ions and due to a nonzero surface charge density at the wall it turns first order or the wall becomes wet at all temperatures $T>T_{t}$.

As discussed in Subsec. IA for the pure solvent it is possible to determine the separatrix between first- and second-order wetting in terms of the surface parameters $h_{1}$ and $g$ only. Accordingly, the phase diagram is of the type shown in Fig. 2(a) of Ref. 12] for $g>0$ and of the type shown there in Fig. 2(b) for $g=0$. On the other hand, for electrolyte solutions this separatrix depends also on the surface charge density, the ionic strength, and the competition between the Debye and the correlation lengths. As mentioned before our approach neglects the interaction between ions so that it can be used only for low ion concentrations, e.g., $I \lesssim 10 \mathrm{mM}$, which corresponds to a Debye length $1 / \kappa \gtrsim 3 \mathrm{~nm}$ in water at room temperature. Thus one typically ends up with case (iii) $(1 / \kappa>2 \xi)$ except in close proximity to the critical point, where one can reach case (iii) $(\xi<1 / \kappa<2 \xi)$ and ultimately case (ii) $(1 / \kappa<\xi)$. Therefore, for $g>0$ the phase diagram for $\sigma \neq 0$ is of the type shown in Fig. 2(a) of Ref. [12], as for the pure solvent case with $g>0$, but the separatrix between first- and second-order wetting is shifted closer to the critical point upon increasing the Debye length, i.e., upon decreasing the ionic strength.

The wetting behavior will be richer if $\xi_{l} \neq \xi_{g}$ (see the discussion below Eq. (35)). In this case, the possible wetting scenarios will depend on the competition between the Debye length $1 / \kappa$, the correlation length $\xi_{g}$ of the gas, and the correlation length $\xi_{l}$ of the liquid. This creates additional cases compared to the ones discussed above (see (ii)-(iii)). Nevertheless, in the present context, far from the critical point case (iii) is still the typical one with the distinction that here $1 /(2 \kappa)$ competes with the maximum of $\xi_{l}$ and $\xi_{g}$.

In the limit $\sigma \rightarrow 0$ one has $a_{I}(T) \rightarrow 0$ so that in this case there is no contribution to the effective interface potential due to the ions. This is due to the fact that within the present theory there are no surfaces fields acting on $\rho_{ \pm}$if $\sigma=0$. For considering instead the limit $I \rightarrow 0$, i.e., $\kappa \rightarrow 0$, in the expression for $a_{I}(T)$ one has to use the saturation value $|\sigma|=\sigma_{\text {sat }}(\kappa \ell)$ given by Eq. (28), which implies $a_{I}(T) \sim \kappa^{3}$. Accordingly, $a_{I}(T) \rightarrow 0$ when $\kappa \rightarrow 0$ so that, as expected, in the limit $I \rightarrow 0$ the pure solvent case is recovered. 


\section{MODEL WITH LONG-RANGED INTERACTIONS}

In this section we consider systems in which the solvent exhibits attractive long-ranged interaction potentials among the solvent particles as well as between the wall and the solvent particles. As in the previous section, we are interested in an analytic expression for the effective interface potential $\omega(\ell)$. Following Ref. [16] we model the attractive part of the pair potential between the solvent particles, as it enters the density functional, as

$$
\bar{w}(r)=\frac{A_{f}}{\left(1+r^{2}\right)^{3}}
$$

with $A_{f}<0$ and the substrate potential as

$$
V(z>0)=-\sum_{i \geq 3} \frac{u_{i}}{z^{i}}
$$

with $u_{3}>0$ corresponding to an asymptotically attractive interaction. The contribution $\sim u_{4}$ is generated, inter alia, by the discrete lattice structure of the substrate or by a thin overlayer [8] and thus it can be tuned. The substrate potential $V(z)$ diverges for $z \rightarrow 0$. Therefore the solvent density $\phi(z)$ must vanish for $z \rightarrow 0$. In the following this effect is taken into account approximately by replacing the short-ranged part of $V(z)$ in Eq. (46) by a hard-wall potential positioned at $z=d_{w}$; the distances $z$ are still measured from $z=0$. (Beyond this sharpkink approximation for the wall-liquid interfacial profile, $d_{w}$ is replaced by the moment $d_{w l}^{(1)}$ (Eq. [D9) of the profile $\phi_{w l}(z)$.) This implies that in the present section the short-ranged description of the surface-fluid interaction given in terms of the surface parameters $h_{1}$ and $g$ in the previous section has to be shifted from $z=0$ to $z=d_{w}$ (see Eq. (10). On the other hand, in order to account for the long-ranged attractive part of $V(z)$ (i.e., for $z \gg d_{w}$ ), here only the first two terms of the sum in Eq. (46) are considered. The functional form in Eq. (45) facilitates to carry out subsequent integrals analytically. These longranged interactions are treated as a perturbation of the grand canonical functional in Eq. (11):

$$
\Omega\left[\phi(\mathbf{r}), \rho_{ \pm}(\mathbf{r})\right]=\Omega_{0}\left[\phi(\mathbf{r}), \rho_{ \pm}(\mathbf{r})\right]+\Delta \Omega[\phi(\mathbf{r})]
$$

where $\Omega_{0}\left[\phi(\mathbf{r}), \rho_{ \pm}(\mathbf{r})\right]$ is given by Eq. (11) and

$$
\begin{aligned}
\Delta \Omega[\phi(\mathbf{r})] & =\frac{1}{2} \int d^{3} r \int d^{3} r^{\prime} \bar{w}\left(\left|\mathbf{r}-\mathbf{r}^{\prime}\right|\right) \phi(\mathbf{r}) \phi(\mathbf{r}) \\
& +\int d^{3} r \rho_{w} V(\mathbf{r}) \phi(\mathbf{r}) .
\end{aligned}
$$

The integrations run over the half space $\{\mathbf{r}=(x, y, z \geq$ $\left.\left.d_{w}\right)\right\}, \bar{w}(r)$ is given by Eq. (45), and $V(\mathbf{r})$ is given by Eq. (46); $\rho_{w}$ is the particle number density of the substrate. Concerning the interaction between the solvent particles, it turns out that it is most suitable captured by the quantity [8],

$$
t(z):=\int_{z}^{\infty} d z^{\prime} \int d^{2} r_{\|}^{\prime} \bar{w}\left(\left(r_{\|}^{\prime 2}+z^{\prime 2}\right)^{1 / 2}\right) .
$$

For large distances and non-retarded van der Waals forces one has

$$
t(z \rightarrow \infty)=-\left(\frac{t_{3}}{z^{3}}+\frac{t_{4}}{z^{4}}+\cdots\right),
$$

which defines the coefficients $t_{3}>0$ and $t_{4}$. For the present model this implies

$$
\begin{gathered}
t_{3}=-\frac{\pi A_{f}}{6} \\
t_{4}=0 .
\end{gathered}
$$

The addition of the long-ranged pair potential between solvent particles (Eq. (45)) modifies the bulk grand canonical potential per $k_{B} T$ of the pure solvent (i.e., $\rho_{ \pm}=0$ ) (see Eq. (2)). Accordingly, in this system the bulk densities $\phi_{l}$ and $\phi_{g}$ minimize the modified bulk grand canonical potential density given by

$$
\begin{aligned}
\beta \Omega_{b, l r}(\phi, \rho=0) & =\phi\left(\ln (\phi)-\beta \tilde{\mu}_{\phi}\right)+(1-\phi) \ln (1-\phi) \\
& +\tilde{\chi}(T) \phi(1-\phi)
\end{aligned}
$$

with the shifted solvent chemical potential $\tilde{\mu}_{\phi}=\mu_{\phi}-$ $\pi^{2} A_{f} / 8$ and the modified Flory-Huggins parameter $\tilde{\chi}(T)=\chi_{S}+\chi_{H} / T-\pi^{2} \beta A_{f} / 8$, i.e., the RPA-like perturbation $\Delta \Omega[\phi(\mathbf{r})]$ in Eq. (48) changes only the enthalpic part of the Flory-Huggins parameter. The binodal $T_{b i, l r}(\phi)$ is again of the form given in Eq. (77) but with $\chi(T)$ replaced by $\tilde{\chi}(T)$. Hence the critical point is located at $\left(\phi_{c}=1 / 2, \tilde{\chi}\left(T_{c}\right)=2\right)$, i.e.,

$$
T_{c}=\frac{\chi_{H}-\pi^{2} A_{f} /\left(8 k_{B}\right)}{2-\chi_{S}} .
$$

The bulk correlation length is now given by (see Appendix B

$$
\frac{1}{\xi^{2}}=\frac{\frac{3}{\tilde{\chi}(T)}\left(\frac{1}{\phi}+\frac{1}{1-\phi}-2 \tilde{\chi}(T)\right)}{1+\frac{\pi^{2} \beta A_{f}}{8 \tilde{\chi}(T)}} .
$$

In a first-order perturbative theory approach (see Appendix $(\mathrm{C})$ the influence of $\Delta \Omega[\phi(\mathbf{r})]$ on the wetting behavior of the electrolyte solution can be determined by inserting into Eq. (47) the solutions $\phi^{(0)}(\mathbf{r})$ and $\rho_{ \pm}^{(0)}(\mathbf{r})$ as obtained from $\Omega_{0}\left[\phi(\mathbf{r}), \rho_{ \pm}(\mathbf{r})\right]$ (see Sec. II). The superscript (0) denotes these solutions as the ones obtained from the unperturbed functional $\Omega_{0}$. 
Following the same procedure as described in Sec. III. i.e., expanding the local part of the grand canonical functional in Eq. (47) around the sharp-kink density profiles in Eqs. (8) and (9), for $\ell \rightarrow \infty$ we obtain the following form for the effective interface potential (see Appendix E):

$$
\begin{aligned}
\beta \omega(\ell \rightarrow \infty) & \simeq \frac{a_{1}(T)}{\ell^{2}}+\frac{b_{1}(T)}{\ell^{3}}+\cdots \\
& +a_{0}(T) \exp (-\ell / \xi)+b_{0}(T) \exp (-2 \ell / \xi) \\
& +a_{I}(T) \exp (-2 \kappa \ell),
\end{aligned}
$$

where ellipses stand for further subdominant terms as powers of $1 / \ell$. As in the absence of long-ranged interactions the ions enter into $\omega(\ell)$ only via the last term. The analytic expressions for the coefficients $a_{1}(T)$ and $b_{1}(T)$ are given in Appendix D $a_{0}(T)$ and $b_{0}(T)$ are given by Eqs. (37) and (38), respectively, and $a_{I}(T)$ is given by Eq. (44). Corrections to the coefficients $a_{0}(T)$ and $b_{0}(T)$ due to the long-ranged interactions (Eqs. (45) and (46) ) are neglected because these long-ranged interactions are treated as a small perturbation to the model with short-ranged interactions only. The sign of the coefficients $a_{1}(T), b_{1}(T), a_{0}(T)$, and $b_{0}(T)$ can change with $T$ while $a_{I}(T)$ is always positive (see Appendix D). As discussed for short-ranged interactions in the previous Sec. III the order of the wetting transition can be inferred from the analysis of these coefficients. They depend on seven parameters: $\chi_{S}, \chi_{H}, A_{f}, u_{3} \rho_{w}, u_{4} \rho_{w}, h_{1}$, and $g$. The value of $\chi_{S}$ is typically much smaller than unity [10] so that we set $\chi_{S}=0$ in the following. Moreover, in the discussion below, the amplitude $A_{f}$ is chosen to be in the range $(0.04-0.15) \times 10^{-19} \mathrm{~J}$, which corresponds to typical strengths of the van der Waals interaction in condensed phases (see Ref. [17]) and $\chi_{H}$ is determined via of Eq. (54) using the critical temperature $T_{c}=647 \mathrm{~K}$ of water. Finally, $u_{3} \rho_{w}$ is fixed by specifying the temperature $T^{\left(a_{1}\right)}$ at which $a_{1}\left(T^{\left(a_{1}\right)}\right)=0$ given by the implicit relation (see Eq. D1) $\phi_{l}\left(T^{\left(a_{1}\right)}\right)=u_{3} \rho_{w} / t_{3}=-6 u_{3} \rho_{w} /\left(\pi A_{f}\right)$ $\left(u_{3}>0, A_{f}<0\right)$; in the case of a critical wetting transition this temperature coincides with the wetting transition temperature $T_{w}=T^{\left(a_{1}\right)}$. With these choices the only remaining free parameters in the following analysis are $u_{4} \rho_{w}, h_{1}$, and $g$. However, their values are constrained by the condition $b_{1}\left(T_{w}\right)>0$ for critical wetting (see Eq. (D2)). Due to the additional presence of the parameters $A_{f}, u_{3} \rho_{w}$, and $u_{4} \rho_{w}$ of the long-ranged interactions, in that case the corresponding discussion is slightly more difficult than the one for short-ranged interactions only as studied in the previous section.

We start this discussion by analyzing the pure solvent case, i.e., $a_{I}=0$. In this case, the necessary conditions for the occurrence of critical wetting are (Eq. (56) and Ref. [8])

$$
a_{1}\left(T_{\mathrm{w}}\right)=0, \quad a_{1}\left(T<T_{\mathrm{w}}\right)<0, \text { and } b_{1}\left(T_{w}\right)>0
$$

i.e., $T^{\left(a_{1}\right)}=T_{w}$, and, as before, one obtains conditions for the parameters of the pair potentials (see Eqs. (D1) and (D2):

$$
\phi_{c} / \rho_{w}<u_{3} / t_{3}<\phi_{l}\left(T_{t}\right) / \rho_{w}
$$

and

$$
\rho_{w} u_{4}-3 t_{3} \phi_{l}\left(T_{w}\right)\left[d_{w}+d_{w l}^{(1)}\right]>0,
$$

with $d_{w l}^{(1)}$ given by Eq. (D9) and $\phi_{l}\left(T_{w}\right)=u_{3} \rho_{w} / t_{3}$.

Although necessary, these conditions are not sufficient for critical wetting to occur. Large negative values of the coefficient $a_{0}(T)$ of the exponentially decaying contribution can still lead to a first-order wetting transition even if $b_{1}\left(T^{\left(a_{1}\right)}\right)>0$. Within the present model one has $a_{0}(T)>0$ for $h_{1} / g>\phi_{l}(T)$ (see Eq. (37)). If $b_{1}\left(T^{\left(a_{1}\right)}\right)<0$ the wetting transition is always first order. However, in the case of a first-order wetting transition all details of $\omega(l)$, and not only its leading contributions, matter for a reliable description of the character of the transition and for determining the corresponding wetting transition temperature. Hence, an asymptotic expansion of $\omega(\ell)$ as in Eq. (56) is not conclusive in the case of first-order wetting.

For wetting of a wall by a one-component fluid with short- and long-ranged interactions and based on a Cahn type theory, in Refs. [18, 19] a wetting scenario has been predicted which involves a succession of two interfacial phase transitions upon increasing $T$. The first of these two transitions is a discontinuous jump between two finite values $\ell_{1}$ and $\ell_{2}>\ell_{1}$ of the film thickness $\ell$ at two-phase coexistence and is referred to as a "thin-thick transition". The second one is the standard second-order wetting transition at $T=T_{w}$. (In Refs. [18, 19] the possibility of a thin-thick transition preceding a first-order wetting transition has not been discussed). This wetting scenario can be explained in terms of the competition between the short- and long-ranged interactions. Such a thin-thick transition precedes the critical wetting transition only if the short-ranged interactions would give rise to a first-order wetting transition in the case that the long-ranged interactions were negligible. Because the present theory involves both short- and long-ranged interactions, the occurrence of this wetting scenario can be checked for the pure solvent case. In this case, the separatrix between first- and second-order wetting is given by Eq. (42) for the model with short-ranged interactions only (e.g., for $g=1$ the transition will be first order in the pure solvent case without long-ranged interaction if $h_{1}>0.49$ ). By choosing a proper set of parameters (see the discussion above) we have been able to observe the occurrence of this two-stage transition for the pure solvent within our model for $\pi^{2} A_{f} \lesssim 0.55 \times 10^{-19} \mathrm{~J}$, $\phi_{l}\left(T_{w}\right)=u_{3} \rho_{w} / t_{3}=0.7, u_{4}=2.3 \times t_{3}, g=1$, and $h_{1}=0.76$, such that the condition for second-order wetting given by Eq. (59) is satisfied.

This thin-thick transition has also been observed for wetting of a wall by a one-component fluid in models with short-ranged interactions only 20 22 and with long-ranged interactions only [23]. Furthermore it has 
been observed experimentally for wetting of hexane on water 24]. In Ref. 20] this thin-thick transition has been observed for a generalization of the Sullivan model 25], in which in addition to the exponentially decaying wall-fluid potential a square-well attraction has been included. A thin-thick transition was also analyzed in Ref. 21] for a Landau theory of wetting which includes an extra surface term $h_{3}(\phi(0))^{3}$ linked to the substrate potential (see Ref. [12] and Eq. (11)). In Ref. [22] it has been shown that the behavior of the model in Ref. 20] can be mapped onto that used in Ref. [21]. With that it turns out that the thin-thick transition predicted in Refs. [20] and 21] involves short-ranged forces only and is due to the competition between two opposing (effective) surface fields at the same surface, one favoring wetting and the other favoring drying. Such a competition between surface fields is not considered here. Therefore within our model a thin-thick transition does not occur in the pure solvent case with short-ranged interactions only (see Sec. III).

The influence of ions and of surface charges on the wetting behavior of electrolytes with solvents governed by short- and long-ranged forces differs qualitatively from the one discussed in Subsec. ПIB, because in this case the leading contributions to $\omega(\ell \rightarrow \infty)$ decay algebraically as function of the film thickness $\ell$. Accordingly, the contribution due to the ions and the charged wall can enter at most as the leading non-algebraic term in the expansion for $\ell \rightarrow \infty$; this is the case if the Debye length $1 / \kappa$ is larger than (twice) the bulk correlation length $\xi$ (see Subsec IIB).

We have considered various parameter sets $\left(h_{1}, g, u_{4}, T^{\left(a_{1}\right)}\right)$ chosen such that the pure solvent with short- and long-ranged interactions near a charge neutral wall (i.e., for $a_{I}(T)=0$ ) exhibits a second-order wetting transition at $T_{w}(I=0, \sigma=0)$ without being preceded by a thin-thick transition (i.e., different from the above scenario). For fixed ionic strength $I \neq 0$ and upon increasing the surface charge density $\sigma$, due to $a_{I}(T) \sim \sigma^{2} / \sqrt{I}(\mathrm{Eq} .44) \omega(\ell)$ rises at finite film thickness $\ell$ to the effect that the wetting transition temperature $T_{w}(I, \sigma)$ decreases for increasing surface charge density $\sigma$ [5]. Moreover, for fixed surface charge density $\sigma$ the wetting transition temperature $T_{w}(I, \sigma)$ decreases upon decreasing the ionic strength $I$ (i.e., increasing the amplitude $\sigma^{2} / \sqrt{I}$ and the Debye length $\left.1 / \kappa \sim 1 / \sqrt{I}\right)[\underline{5}]$. In addition, the positive and monotonically decreasing (as a function of increasing $\ell$ ) contribution $a_{I}(T) \exp (-2 \kappa \ell)$ to $\omega(\ell)$ does lead to a thin-thick transition preceding the critical wetting transition which is absent without ions. Figure 1 shows the curves for $\omega(\ell)$ corresponding to the temperatures $T_{1}=0.918 \times T_{c}, T_{2}=0.919 \times T_{c}$, $T_{3}=0.92 \times T_{c}, T_{4}=0.932 \times T_{c}$, and $T_{w}=0.944 \times T_{c}$ with $T_{1}<T_{2} \lesssim T_{t-t, w}<T_{3}<T_{4}<T_{w}$, i.e., the thin- $t$ hick transition occurs in between the temperatures $T_{2}$ and $T_{3}$, whereas the critical wetting transition takes place at the wetting temperature $T_{w}$.

However, in the case that the pure solvent exhibits a second-order wetting transition, which is preceded by a thin-thick wetting transition, the effect of the term due to the ions and to the surface charge density $\left(a_{I}(T) \neq 0\right)$, in the case $1 / \kappa>2 \xi$, is to decrease the thin-thick wetting transition temperature $T_{t-t, w}$ and to increase the value of the jump in film thickness.

The case of $a_{I}(T) \neq 0$ for a system in which a pure solvent with short- and long-ranged interactions near a charge neutral wall exhibits a first-order wetting transition is not discussed here, because within the present approach only the leading contributions of the effective interface potential for $\ell \rightarrow \infty$ are analytically accessible (see Eq. (56) ) and reliable knowledge of the behavior of $\omega(\ell)$ for small $\ell$, which is particularly important for first-order wetting transitions, is lacking. Therefore, in order to be able to analyze the effect of the ions and of the surface charge density on solvents which without ions exhibit first-order wetting transitions, more details of the effective interface potential are needed.

The thin-thick wetting transition at two-phase coexistence, which precedes a standard second-order wetting scenario, has been discussed in the context of wetting in electrolytes in Ref. [2] for a model of an ionic solution close to a charged wall in which the solvent-solvent and solvent-wall interactions are short-ranged only and the contribution of the ions to the effective interface potential is calculated by solving the full Poisson-Boltzmann equation instead of the linearized one as in the present study (see Sec. II). The thin-thick transition in Ref. [2] occurs in a restricted region of the parameter space, provided that the transition in the pure solvent is first order and that $1 / \kappa<2 \xi$, i.e, for large ionic strength.

In contrast, within the present approach the combined presence of short- and long-ranged interactions is taken into account. As discussed above for the case of a pure solvent with short- and long-ranged interactions, a thin-thick transition will precede a long-ranged critical wetting transition only if the short-ranged interactions alone would give rise to a first-order wetting transition in the case that the long-ranged interactions were negligible [18, 19]. This is precisely the case we encounter in the present context for the electrolyte solution when solvent-solvent and solvent-wall long-ranged interactions are taken into account: In the absence of these long-ranged interactions the transition is first-order if $1 / \kappa>2 \xi$ (see Subsec. IIB), such that $\ell$ jumps from $\ell_{1}$ to $\ell_{2}=\infty$ (see Fig. 11). Once the long-ranged interactions are taken into account they block the jump of $\ell$ to $\ell_{2}=\infty$ and limit this jump to one with a finite value $\ell_{2}<\infty$. Once $\ell$ has reached the value $\ell_{2}$ a further increase in temperature leads to the unfolding of the standard wetting scenario under the aegis of long-ranged interactions at $T_{w}>T_{t-t, w}$. Therefore, the thin-thick wetting transition is the remnant of the first-order wetting transition that would occur in the electrolyte solution if the longranged solvent-solvent and solvent-wall interactions were negligible (see Subsec. II B).

We briefly give the main points of the literature and 



FIG. 1. Effective interface potential $\omega(\ell)$ for systems governed by short- and long-ranged interactions as function of the thickness $\ell$ of the liquid film at gas-liquid coexistence in the presence of ions for the case that the pure, i.e., salt-free, solvent exhibits a critical wetting transition (without being preceded by a thin-thick transition). The parameters used are $A_{f} / k_{B}=-1013 \mathrm{~K}$, $u_{3}=0.7 \times t_{3}$ (i.e., $\left.\phi_{l}\left(T^{\left(a_{1}\right)}=T_{w}\right)=0.7\right), u_{4}=2.28 \times t_{3}, g=1, h_{1}=0.76 \times g, I=1 \mathrm{mM}$, and $\sigma=0.1 \mu \mathrm{C} / \mathrm{cm}^{2}$ (see main text). The effective interface potential $\omega(\ell)$ has two local minima (at $\ell_{1}(T)$ (see (a) and (b)) and $\ell_{2}(T)\left(\right.$ see $(\mathrm{c})$ ) with $\ell_{1}<\ell_{2}<\infty$ ), one of the two being the global one at a given temperature (see (a)). They have the same depth at $T=T_{t-t, w} \approx 0.919 \times T_{c}$ (not apparently visible). For $T>T_{t-t, w}$ the film thickness $\ell_{2}(T)$ is the global minimum and diverges continuously $1 /\left(T_{w}-T\right)$ as $T \rightarrow T_{w} \approx 0.944 \times T_{c}$ (see $(\mathrm{c})$ ). The global minimum $l_{0}(T)$ as a function of temperature is plotted in (d). At $T_{t-t, w}$ the film thickness exhibits a finite jump and subsequently diverges smoothly for $T \nearrow T_{w}$. Accordingly, the system undergoes a thin-thick wetting transition at $T_{t-t, w}$, followed by a continuous one at $T_{w}$. Five different temperatures, $T_{1} \approx 0.918 \times T_{c}$, $T_{2} \approx 0.919 \times T_{c}, T_{3} \approx 0.92 \times T_{c}, T_{4}=0.932 \times T_{c}$ and $T_{w}$ are displayed in (a), (b), and (c) (using a common color code) with $T_{1}<T_{2} \lesssim T_{t-t, w}<T_{3}<T_{4}<T_{w}$. (Note the different scales of the axes.) The film thickness $\ell$ is measured in units of $a$ such that $a^{3}$ is the volume of a solvent particle. Densities are measured in units of $a^{3}$.

of our results.

(I) If in the pure solvent short-ranged interactions favor first-order wetting but additional long-ranged interactions produce second-order wetting, one finds a thin-thick transition followed by the continuous wetting transition [18, 19]. We confirmed this behavior for our model, which allows for the thin-thick transition only via the competition between short- and long-ranged interactions.

(II) A thin-thick transition can be observed in the pure solvent even if there are only short-ranged [20 22 or only long-ranged 23] interactions.
(III) We have considered a solvent with short- and longranged interactions which exhibits a second-order wetting transition without being preceded by a thin-thick transition. Adding ions renders such a short-ranged contribution to the effective interface potential that the resulting effective short-ranged interactions favor first-order wetting. This leads to a thin-thick transition preceding the continuous long-range type wetting transition. This mechanism is analogous to the one in (I).

(IV) If the solvent with short- and long-ranged interactions undergoes a continuous wetting transition, which is preceded by a thin-thick transition, 
adding ions decreases the transition temperature of the latter and increases the jump in film thickness.

(V) If the pure solvent is governed by short-ranged interactions only and exhibits a first-order wetting transition, adding ions can lead to a continuous wetting transition preceded by a thin-thick transition, provided that $1 / \kappa<2 \xi$.

(VI) If the solvent is governed by short-ranged interactions only, adding ions renders a first-order wetting transition for $1 / \kappa>2 \xi$. Adding further longranged interactions, which favor continuous wetting, renders a second-order wetting transition of the long-range type, preceded by a thin-thick transition.

\section{CONCLUSIONS AND SUMMARY}

We have implemented an improvement over the approximation of step-like varying density profiles in order to derive analytic expressions for the effective interface potential $\omega(\ell)$ of electrolyte solutions near charged walls. This approach consists of performing a Taylor expansion up to second order of the local part of the grand canonical density functional around piecewise constant density profiles of the solvent and of the ions. The resulting mean-field expressions for the effective interface potential allow one to predict general trends for the wetting behavior of electrolyte solutions in terms of the relevant system parameters such as the ionic strength and the surface charge density.

The present analysis, which is valid in the case of low ion density $I$, shows that in the case of shortranged solvent-solvent and solvent-wall interactions wetting transitions in the presence of electrostatic interactions are typically first order. This result can be explained in terms of the competition between the two characteristic length scales in the system, i.e., the bulk correlation length $\xi$ in the wetting liquid phase and the Debye length $1 / \kappa$. If $1 / \kappa>2 \xi$, which is typically the case for dilute electrolyte solutions away from (bulk) critical points, a wetting transition at two-phase coexistence will be always first order irrespective of its order in the pure, i.e., salt-free, solvent. First-order wetting transitions in electrolyte solutions with solvent interactions being shortranged only have been observed in previous studies, too [1 5]. It is the merit of the present analysis of the effective interface potential to provide a transparent rationale for the pre-eminence of first-order wetting in electrolyte solutions in terms of competing length scales. Moreover, if in those systems in addition long-ranged solvent-solvent and solvent-wall interactions, which favor a critical wetting transition, are present, our analysis reveals the possibility of a wetting scenario which actually corresponds to a sequence of two wetting transitions: first an electrostatically induced (i.e., $1 / \kappa>2 \xi$ ) discontinuous jump between two finite wetting film thicknesses which upon raising the temperature is followed by a continuous divergence of the wetting film thickness $\ell$ (see Fig. 1).

Within the present approach, in the case of shortranged interactions the analytic expressions for the coefficients of the exponential terms in the effective interface potential $\omega(\ell)$ (Eq. (35) ) are simple and the necessary conditions for first- and second-order wetting can be translated explicitly into conditions for the parameters of the interaction potentials. However, in the case of additional long-ranged interactions extra parameters make such a kind of translation more difficult. Nevertheless, by choosing a set of parameters for the interaction potentials based on actual values for the Hamaker constant and the critical temperature and by fulfilling corresponding necessary conditions for the occurrence of second-order wetting in the pure solvent (formulated in terms of the coefficients $a_{1}(T)$ and $b_{1}(T)$ (Eqs. (56), (D1), and (D2) )), we have been able to analyze the effect of ions and of the surface charge density of the confining wall on the wetting behavior. We have found that if the pure solvent exhibits a second-order wetting transition governed asymptotically by long-ranged interactions, adding ions typically introduces a thin-thick transition which precedes the ultimate continuous wetting transition (see Fig. 1). We have been able to put the occurrence of such a thin-thick wetting transition at gas-liquid coexistence into the context of the literature, which discusses such a transition as the result of the interplay between short- and long-ranged interactions. Here the corresponding short-ranged effective interactions relevant for that are provided by the ions.

\section{Appendix A: Double parabola approximation for the pure solvent}

The double parabola approximation (DPA) has been widely used in the context of wetting phenomena in order to obtain analytically tractable density functionals [27 34]. Within this approximations, the grand canonical functional for the pure solvent, i.e., $\rho_{ \pm}=0$ and $\mathbf{D}\left(\mathbf{r},\left[\rho_{ \pm}\right]\right)=0$ (see Eq. (1) ) is given by

$$
\begin{aligned}
\frac{\beta \Omega_{D P A}[\phi(z)]}{A} & =\int d z\left\{F_{D P A}(\phi(z))+\frac{\chi(T)}{6}\left(\frac{d \phi(z)}{d z}\right)^{2}\right. \\
& \left.-\phi(z) \beta \mu_{\phi}\right\} \\
& -\beta h_{1} \phi(z=0)+\beta \frac{g}{2}(\phi(z=0))^{2}
\end{aligned}
$$

with

$$
F_{D P A}(\phi)=C(T) \begin{cases}\left(\phi-\phi_{l}(T)\right)^{2}, & \phi>\frac{1}{2}\left(\phi_{l}(T)+\phi_{g}(T)\right) \\ \left(\phi-\phi_{g}(T)\right)^{2}, & \phi<\frac{1}{2}\left(\phi_{l}(T)+\phi_{g}(T)\right),\end{cases}
$$

where $\phi_{l}(T)$ and $\phi_{g}(T)$ are, respectively, the (temperature dependent) liquid and gas bulk densities at coexistence, and $C(T)$ is fixed later in order to render the bulk 
correlation length. Upon construction, the DPA requires an underlying theory which provides expressions for the bulk densities and the curvature of the local free energy density at coexistence [27 34]. For brevity we shall refrain from indicating the temperature dependence in our notation. Within this approach, for a given profile $\phi(z)$ the assigned film thickness $\ell_{D P A}$ is defined as

$$
\phi\left(z=\ell_{D P A}\right)=\frac{1}{2}\left(\phi_{l}+\phi_{g}\right)
$$

Minimization of the functional in Eq. (A1) leads to

$$
\phi_{D P A}^{\prime \prime}(z)= \begin{cases}\frac{6 C}{\chi}\left(\phi_{D P A}(z)-\phi_{l}\right)-\beta \mu_{\phi}, & \phi_{D P A}(z)>\frac{1}{2}\left(\phi_{l}+\phi_{g}\right) \\ \frac{6 C}{\chi}\left(\phi_{D P A}(z)-\phi_{g}\right)-\beta \mu_{\phi}, & \phi_{D P A}(z)<\frac{1}{2}\left(\phi_{l}+\phi_{g}\right)\end{cases}
$$

with the boundary conditions

$$
\begin{aligned}
\frac{\chi}{3} \phi_{D P A}^{\prime}(0) & =\beta\left(-h_{1}+g \phi_{D P A}(0)\right), \\
\phi_{D P A}\left(\ell_{D P A}\right) & =\frac{1}{2}\left(\phi_{l}+\phi_{g}\right), \\
\phi_{D P A}(\infty) & =\phi_{g} .
\end{aligned}
$$

At two-phase coexistence $\mu_{\phi}=0$ (as for the pure solvent case, i.e., $\rho_{ \pm}=0$, in the present model given by Eq. (2)) and Eq. A4 reduces to

$\phi_{D P A}^{\prime \prime}(z)= \begin{cases}\frac{6 C}{\chi}\left(\phi_{D P A}(z)-\phi_{l}\right), & \phi_{D P A}(z)>\frac{1}{2}\left(\phi_{l}+\phi_{g}\right) \\ \frac{6 C}{\chi}\left(\phi_{D P A}(z)-\phi_{g}\right), & \phi_{D P A}(z)<\frac{1}{2}\left(\phi_{l}+\phi_{g}\right) .\end{cases}$

Comparison with Eq. (15) leads to $C=\chi /\left(6 \xi^{2}\right)$. Equation (A6) together with the boundary conditions in Eq. (A5) yields

$\phi_{D P A}(z)= \begin{cases}C_{1} \exp \left(\frac{z}{\xi}\right)+C_{2} \exp \left(-\frac{z}{\xi}\right)+\phi_{l}, & 0 \leq z \leq \ell_{\mathrm{DPA}} \\ C_{3} \exp \left(-\frac{z}{\xi}\right)+\phi_{g}, & z \geq \ell_{\mathrm{DPA}}\end{cases}$

where

$$
\begin{aligned}
C_{1} & =\frac{\frac{\phi_{g}-\phi_{l}}{2}\left(\beta g+\frac{\chi}{3 \xi}\right)-\beta\left(h_{1}-g \phi_{l}\right) \exp \left(-\ell_{\mathrm{DPA}} / \xi\right)}{\left(\beta g+\frac{\chi}{3 \xi}\right) \exp \left(\ell_{\mathrm{DPA}} / \xi\right)+\left(\frac{\chi}{3 \xi}-\beta g\right) \exp \left(-\ell_{\mathrm{DPA}} / \xi\right)}, \\
C_{2} & =\frac{\frac{\phi_{g}-\phi_{l}}{2}\left(\frac{\chi}{3 \xi}-\beta g\right)+\beta\left(h_{1}-g \phi_{l}\right) \exp \left(\ell_{\mathrm{DPA}} / \xi\right)}{\left(\beta g+\frac{\chi}{3 \xi}\right) \exp \left(\ell_{\mathrm{DPA}} / \xi\right)+\left(\frac{\chi}{3 \xi}-\beta g\right) \exp \left(-\ell_{\mathrm{DPA}} / \xi\right)} \\
C_{3} & =\frac{\phi_{l}-\phi_{g}}{2} \exp \left(\ell_{\mathrm{DPA}} / \xi\right)
\end{aligned}
$$

The comparison between Eqs. (22) and (A7) shows that the coefficients $\hat{A}_{l}, \hat{B}_{l}$, and $\hat{B}_{g}$ there play the same role as the coefficients $C_{1}, C_{2}$, and $C_{3}$, respectively, here. At coexistence and for $\ell=\ell_{\mathrm{DPA}}$ in Eq. (29) one obtains

$$
\begin{aligned}
& C_{1}=A_{l}\left(1+\mathcal{O}\left(\exp \left(-\ell_{\mathrm{DPA}} / \xi\right)\right)\right), \\
& C_{2}=B_{l}\left(1+\mathcal{O}\left(\exp \left(-\ell_{\mathrm{DPA}} / \xi\right)\right)\right) \\
& C_{3}=B_{g}\left(1+\mathcal{O}\left(\exp \left(-\ell_{\mathrm{DPA}} / \xi\right)\right)\right),
\end{aligned}
$$

i.e., the relative difference between the coefficients of the profiles in Eq. (22) and in Eq. (A7) is exponentially small for film thicknesses $\ell=\ell_{\mathrm{DPA}} \gg \xi$ large compared to the bulk correlation length.

\section{Appendix B: Bulk correlation length of the pure solvent}

In the case of a bulk pure solvent $(I=0)$, the density functional $\Omega_{0}$ given by Eq. (11) reduces to

$$
\begin{aligned}
\beta \Omega_{0}^{(p)}[\phi(\mathbf{r})] & =\int d^{3} r\left\{\phi(\mathbf{r})\left(\ln (\phi(\mathbf{r}))-\beta \mu_{\phi}\right)\right. \\
& +(1-\phi(\mathbf{r})) \ln (1-\phi(\mathbf{r})) \\
& \left.+\chi(T) \phi(\mathbf{r})(1-\phi(\mathbf{r}))+\frac{\chi(T)}{6}(\nabla \phi(\mathbf{r}))^{2}\right\} .
\end{aligned}
$$

If we consider a spatially uniform equilibrium state $\phi$, the corresponding two-point correlation function $G(r)=$ $\phi^{2} h(r)=\phi^{2}(g(r)-1)$ is obtained from $G(r)=$ $G(\mathbf{r}, \mathbf{0})$, with the inverse $G^{-1}\left(\mathbf{r}, \mathbf{r}^{\prime}\right)=\frac{\delta^{2} \Omega}{\delta \phi(\mathbf{r}) \delta \phi\left(\mathbf{r}^{\prime}\right)}$, where $\int d^{3} r^{\prime \prime} G\left(\mathbf{r}, \mathbf{r}^{\prime \prime}\right) G^{-1}\left(\mathbf{r}^{\prime \prime}, \mathbf{r}^{\prime}\right)=\delta\left(\mathbf{r}-\mathbf{r}^{\prime}\right)$ [6]. From Eq. (B1) one obtains

$$
\begin{aligned}
G^{-1}\left(\mathbf{r}, \mathbf{r}^{\prime}\right)=\delta\left(\mathbf{r}-\mathbf{r}^{\prime}\right)\left(\frac{1}{\phi}\right. & \left.+\frac{1}{1-\phi}-2 \chi(T)\right) \\
& -\frac{\chi(T)}{3} \nabla^{2} \delta\left(\mathbf{r}-\mathbf{r}^{\prime}\right) .
\end{aligned}
$$

The corresponding Fourier transform, $\hat{G}^{-1}(k)=$ $\int d^{3} r G^{-1}\left(\mathbf{r}, \mathbf{r}^{\prime}\right) e^{-i \mathbf{k} \cdot\left(\mathbf{r}-\mathbf{r}^{\prime}\right)}$ with dimensionless $\mathbf{k}$, is given by

$$
\hat{G}^{-1}(k)=\frac{\chi(T)}{3}\left(\frac{1}{\xi^{2}}+k^{2}\right),
$$

where Eq. (16) has been used. $\hat{G}(k)$, which is proportional to the static structure factor, can be written in the form

$$
\hat{G}(k)=\frac{\frac{3 \xi^{2}}{\chi(T)}}{1+(k \xi)^{2}}=\frac{\hat{G}(0)}{1+(k \xi)^{2}} .
$$


This allows one to identify $\xi$ with the bulk correlation length. For a discussion of the structure in the presence of ions (Eq. (1)) see Ref. [9].

In a similar way for the case with long-ranged interaction between solvent particles (see Eq. (477)

$$
\begin{aligned}
G^{-1}\left(\mathbf{r}, \mathbf{r}^{\prime}\right)=\delta\left(\mathbf{r}-\mathbf{r}^{\prime}\right)\left(\frac{1}{\phi}+\right. & \left.\frac{1}{1-\phi}-2 \chi(T)+\frac{\beta \pi^{2} A_{f}}{4}\right) \\
& -\frac{\chi(T)}{3} \nabla^{2} \delta\left(\mathbf{r}-\mathbf{r}^{\prime}\right) .
\end{aligned}
$$

Therefore the bulk correlation length $\xi$ for this case is given by

$$
\begin{aligned}
\frac{1}{\xi^{2}} & =\frac{3}{\chi(T)}\left(\frac{1}{\phi}+\frac{1}{1-\phi}-2 \chi(T)+\frac{\pi^{2} \beta A_{f}}{4}\right) \\
= & \frac{\frac{3}{\tilde{\chi}(T)}\left(\frac{1}{\phi}+\frac{1}{1-\phi}-2 \tilde{\chi}(T)\right)}{1+\frac{\pi^{2} \beta A_{f}}{8 \tilde{\chi}(T)}}
\end{aligned}
$$

with $\tilde{\chi}(T)=\chi(T)-\pi^{2} \beta A_{f} / 8$.

\section{Appendix C: First-order perturbation theory for including the long-ranged interactions}

The total grand canonical functional is given by

$$
\Omega\left[\phi(\mathbf{r}), \rho_{ \pm}(\mathbf{r})\right]=\Omega_{0}\left[\phi(\mathbf{r}), \rho_{ \pm}(\mathbf{r})\right]+\Delta \Omega[\phi(\mathbf{r})]
$$

with $\Omega_{0}\left[\phi(\mathbf{r}), \rho_{ \pm}(\mathbf{r})\right]$ given by Eq. (11) whereas $\Delta \Omega[\phi(\mathbf{r})]$ is given by Eq. (48) and depends only on $\phi(\mathbf{r})$. We choose a dimensionless coupling parameter $\lambda \in[0,1]$ such that for $\lambda=0$ the perturbation $\Delta \Omega$ is absent and for $\lambda=1$ the perturbation is fully present. The perturbed grand canonical functional is

$$
\Omega_{\lambda}\left[\phi(\mathbf{r}), \rho_{ \pm}(\mathbf{r})\right]=\Omega_{0}\left[\phi(\mathbf{r}), \rho_{ \pm}(\mathbf{r})\right]+\lambda \Delta \Omega[\phi(\mathbf{r})]
$$

where $\lambda$ acts as an amplitude multiplying both $\bar{w}\left(\left|\mathbf{r}-\mathbf{r}^{\prime}\right|\right)$ and $V(\mathbf{r})$ (see Eq. (48)). The equilibrium densities $\phi_{\lambda}(\mathbf{r})$ and $\rho_{ \pm, \lambda}(\mathbf{r})$ minimize $\Omega_{\lambda}$ :

$$
\frac{\delta \Omega_{\lambda}}{\delta \phi(\mathbf{r})}\left[\phi_{\lambda}(\mathbf{r}), \rho_{ \pm, \lambda}(\mathbf{r})\right]=0, \quad \frac{\delta \Omega_{\lambda}}{\delta \rho_{ \pm}(\mathbf{r})}\left[\phi_{\lambda}(\mathbf{r}), \rho_{ \pm, \lambda}(\mathbf{r})\right]=0 .
$$

Furthermore, the equilibrium densities $\phi^{(0)}(\mathbf{r}) \equiv \phi_{\lambda=0}(\mathbf{r})$ and $\rho_{ \pm}^{(0)}(\mathbf{r}) \equiv \rho_{ \pm, \lambda=0}(\mathbf{r})$ which minimize $\Omega_{0}\left[\phi(\mathbf{r}), \rho_{ \pm}(\mathbf{r})\right]$ are known (see Sec. III).

In order to proceed we write the equilibrium densities $\phi_{\lambda}(\mathbf{r})$ and $\rho_{ \pm, \lambda}(\mathbf{r})$ as power series in terms of $\lambda$,

$$
\begin{array}{r}
\phi_{\lambda}(\mathbf{r})=\sum_{n=0}^{\infty} \lambda^{n} \phi^{(n)}(\mathbf{r})=\phi^{(0)}(\mathbf{r})+\mathcal{O}(\lambda), \\
\rho_{ \pm, \lambda}(\mathbf{r})=\sum_{n=0}^{\infty} \lambda^{n} \rho_{ \pm}^{(n)}(\mathbf{r})=\rho_{ \pm}^{(0)}(\mathbf{r})+\mathcal{O}(\lambda),
\end{array}
$$

and perform a functional Taylor expansion of the grand canonical potential $\Omega_{\lambda}\left[\phi_{\lambda}(\mathbf{r}), \rho_{ \pm, \lambda}(\mathbf{r})\right]$ around $\phi^{(0)}(\mathbf{r})$, $\rho_{ \pm}^{(0)}(\mathbf{r})$ :

$$
\begin{aligned}
\Omega_{\lambda}\left[\phi_{\lambda}(\mathbf{r}), \rho_{ \pm, \lambda}(\mathbf{r})\right] & =\Omega_{0}\left[\phi^{(0)}(\mathbf{r})+\left(\phi_{\lambda}(\mathbf{r})-\phi^{(0)}(\mathbf{r})\right), \rho_{ \pm}^{(0)}(\mathbf{r})+\left(\rho_{ \pm, \lambda}(\mathbf{r})-\rho_{ \pm}^{(0)}(\mathbf{r})\right)\right] \\
& +\lambda \Delta \Omega\left[\phi^{(0)}(\mathbf{r})+\left(\phi_{\lambda}(\mathbf{r})-\phi^{(0)}(\mathbf{r})\right)\right] \\
& =\Omega_{0}\left[\phi^{(0)}(\mathbf{r}), \rho_{ \pm}^{(0)}(\mathbf{r})\right]+\lambda \Delta \Omega\left[\phi^{(0)}(\mathbf{r})\right] \\
& +\int d^{3} r\left\{\left.\frac{\delta \Omega_{0}\left[\phi(\mathbf{r}), \rho_{ \pm}(\mathbf{r})\right]}{\delta \phi(\mathbf{r})}\right|_{\phi^{(0)}(\mathbf{r}), \rho_{ \pm}^{(0)}(\mathbf{r})}\left(\phi_{\lambda}(\mathbf{r})-\phi^{(0)}(\mathbf{r})\right)\right. \\
& +\left.\frac{\delta \Omega_{0}\left[\phi(\mathbf{r}), \rho_{ \pm}(\mathbf{r})\right]}{\delta \rho_{+}(\mathbf{r})}\right|_{\phi^{(0)}(\mathbf{r}), \rho_{ \pm}^{(0)}(\mathbf{r})}\left(\rho_{+, \lambda}(\mathbf{r})-\rho_{+}^{(0)}(\mathbf{r})\right) \\
& \left.+\left.\frac{\delta \Omega_{0}\left[\phi(\mathbf{r}), \rho_{ \pm}(\mathbf{r})\right]}{\delta \rho_{-}(\mathbf{r})}\right|_{\phi^{(0)}(\mathbf{r}), \rho_{ \pm}^{(0)}(\mathbf{r})}\left(\rho_{-, \lambda}(\mathbf{r})-\rho_{-}^{(0)}(\mathbf{r})\right)\right\}+\mathcal{O}\left(\lambda^{2}\right) \\
& =\Omega_{0}\left[\phi^{(0)}(\mathbf{r}), \rho_{ \pm}^{(0)}(\mathbf{r})\right]+\lambda \Delta \Omega\left[\phi^{(0)}(\mathbf{r})\right]+\mathcal{O}\left(\lambda^{2}\right) \\
& =\Omega_{\lambda}\left[\phi^{(0)}(\mathbf{r}), \rho_{ \pm}^{(0)}(\mathbf{r})\right]+\mathcal{O}\left(\lambda^{2}\right)
\end{aligned}
$$

with $\phi_{\lambda}(\mathbf{r})-\phi^{(0)}(\mathbf{r})=\mathcal{O}(\lambda)$ and $\rho_{ \pm, \lambda}(\mathbf{r})-\rho_{ \pm}^{(0)}(\mathbf{r})=$ $\mathcal{O}(\lambda)$ (see Eq. (C4)). In Eq. (C5) the first deriva- tives vanish because $\phi^{(0)}$ and $\rho_{ \pm}^{(0)}$ minimize $\Omega_{0}$. Hence $\Omega_{\lambda=1}\left[\phi_{\lambda=1}(\mathbf{r}), \rho_{ \pm, \lambda=1}(\mathbf{r})\right] \approx \Omega_{\lambda=1}\left[\phi^{(0)}(\mathbf{r}), \rho_{ \pm}^{(0)}(\mathbf{r})\right]=$ 
$\Omega\left[\phi^{(0)}(\mathbf{r}), \rho_{ \pm}^{(0)}(\mathbf{r})\right]$ up to second order in $\lambda$.

\section{Appendix D: Coefficients for the effective interface potential in the presence of long-ranged interactions}

The effective interface potential for the model with long-ranged interaction (Sec. III) is calculated by following the procedure described in Sec. III The double integrals in Eq. (48) have been evaluated by performing an asymptotic expansion for $\ell \rightarrow \infty$. The analytic expressions for the coefficients in Eq. (56) are given by (see Appendix E)

$$
a_{1}(T)=\frac{\left(\phi_{l}-\phi_{g}\right)}{2} \beta\left(u_{3} \rho_{w}-t_{3} \phi_{l}\right)
$$

and

$$
\begin{aligned}
b_{1}(T) & =\frac{\beta\left(\phi_{l}-\phi_{g}\right)}{3}\left(\rho_{w} u_{4}-3 t_{3} \phi_{l} d_{w}\right. \\
& \left.+3 \xi t_{3} \exp \left(-\frac{d_{w}}{\xi}\right)\left(\frac{\beta\left(h_{1}-g \phi_{l}\right)}{\beta g+\frac{\chi(T)}{3 \xi}}\right)\right) .
\end{aligned}
$$

The coefficients $a_{1}$ and $b_{1}$ can be compared with the general expressions obtained in Ref. [26] within a systematic study of wetting transitions of a simple onecomponent fluid, inter alia including the presence of van der Waals tails. There the effective interface potential is expressed in terms of the interfacial profiles which emerge as a consequence of wetting phenomena, i.e., the wallliquid and the free liquid-gas interface for wetting of the wall-gas interface. Within that approach the effective interface potential at coexistence is given by

$$
\omega(\ell)=\sum_{k=2}^{4} \bar{a}_{k} \ell^{-k}+\mathcal{O}\left(\ell^{-5} \ln \ell\right)
$$

where

$$
\begin{aligned}
& \bar{a}_{2}=\frac{1}{2}\left(\phi_{l}-\phi_{g}\right)\left(\rho_{w} u_{3}-\phi_{l} t_{3}\right), \\
& \bar{a}_{3}=\bar{a}_{3}^{(0)}-2 \bar{a}_{2} d_{l g}^{(1)},
\end{aligned}
$$

and

$$
\bar{a}_{4}=\bar{a}_{4}^{(0)}-3 a_{3} d_{l g}^{(1)}+3 \bar{a}_{2}\left[d_{l g}^{(2)}-2\left(d_{l g}^{(1)}\right)^{2}\right],
$$

with

$$
\left.\bar{a}_{3}^{(0)}=\frac{1}{3}\left(\phi_{l}-\phi_{g}\right)\right)\left[\rho_{w} u_{4}-\phi_{l}\left(t_{4}+3 t_{3} d_{w l}^{(1)}\right)\right]
$$

and

$$
\left.\bar{a}_{4}^{(0)}=\frac{1}{4}\left(\phi_{l}-\phi_{g}\right)\right)\left[\rho_{w} u_{5}-\phi_{l}\left(t_{5}+4 t_{4} d_{w l}^{(1)}+6 t_{3} d_{w l}^{(2)}\right)\right] .
$$

The coefficients $t_{3}, t_{4}$ for the present model are given by Eqs. (51) and (52); $d_{w l}^{(i)}$ and $d_{l g}^{(i)}$ are moments of the wallliquid interface profile $\phi_{w l}(z)$ and of the free liquid-gas interface profile $\phi_{l g}(z)$, respectively:

$$
d_{w l}^{(i)}=i \int_{0}^{\infty} d z z^{i-1}\left[1-\frac{\phi_{w l}(z)}{\phi_{l}}\right], \quad i=1,2,
$$

and

$$
d_{l g}^{(i)}=\frac{i}{\phi_{l}-\phi_{g}} \int_{-\infty}^{\infty} d z z^{i-1}\left[\phi_{l g}(z)-\phi_{l g}^{s k}(z)\right], \quad i=1,2 .
$$

The wall-liquid and the free liquid-gas interface profile can be calculated within our approach by following a procedure analogous to the one described in Sec. [I] To this end, in the case of the wall-liquid interface for the pure solvent, the Taylor expansion up to second order of the local part of the functional in Eq. (1) with $\rho_{ \pm}=0$ and $\mathbf{D}=0$, is performed about $\phi_{w l}(z)=\phi_{l} \Theta\left(z-d_{w}\right)$ for $z \geq 0$ where $\Theta(x)$ is the Heaviside function. This leads to the wall-liquid density profile

$$
\phi_{w l}(z)=\left(\phi_{l}+\frac{\beta\left(h_{1}-g \phi_{l}\right)}{\beta g+\frac{\chi(T)}{3 \xi}} \exp (-z / \xi)\right) \Theta\left(z-d_{w}\right) .
$$

Within the approximation discussed in Appendix C, this expression, obtained from minimizing $\Omega_{0}$ and shifting by $d_{w}$, is inserting into the general expression in Eq. (D9) corresponding to $\Delta \Omega$ and yields

$$
d_{w l}^{(1)}=d_{w}-\frac{\beta \xi\left(h_{1}-g \phi_{l}\right)}{\phi_{l}\left(\beta g+\frac{\chi(T)}{3 \xi}\right)} \exp \left(-d_{w} / \xi\right) .
$$

As expected, Eq. (D12) respects the expected property that in the sharp-kink limit (i.e, vanishing interfacial width $\xi) d_{w l}^{(1)}$ reduces to $d_{w}$. With this result Eq. (D2) can be rewritten as $b_{1}(T)=(\beta / 3)\left(\phi_{l}-\right.$ $\left.\phi_{g}\right)\left(\rho_{w} u_{4}-3 t_{3} \phi_{l} d_{w l}^{(1)}\right)$. For the free liquid-gas interface, we consider the functional in Eq. (B1). All integrals extend over a macroscopic volume. We impose the boundary conditions $\phi_{l g}(z \rightarrow-\infty)=\phi_{l}$ and $\phi_{l g}(z \rightarrow \infty)=\phi_{g}$. Accordingly, the Taylor expansion up to second order of the local part of the functional is performed about the $s$ harp- $k$ ink profile

$$
\phi_{l g}^{s k}(z)= \begin{cases}\phi_{l}, & z<0 \\ \phi_{g}, & z>0 .\end{cases}
$$

The resulting liquid-gas density profile based on $\Omega_{0}$ is

$$
\phi_{l g}(z)= \begin{cases}\phi_{l}+\frac{\phi_{g}-\phi_{l}}{2} \exp (z / \xi), & z<0 \\ \phi_{g}+\frac{\phi_{l}-\phi_{g}}{2} \exp (-z / \xi), & z>0\end{cases}
$$

Again, within the approximation discussed in Appendix C. this profile stemming from $\Omega_{0}$ is inserted into the general expression in Eq. (D10), which is based on $\Delta \Omega$, and renders

$$
d_{l g}^{(1)}=0
$$


Inserting Eqs. (51), (52), (D12), and (D15) into Eqs. (D4) and (D5) one obtains (with $t_{4}=0$ )

$$
\beta \bar{a}_{2}=a_{1} \text { and } \beta \bar{a}_{3}=b_{1} .
$$

This leads to the satisfactory statement that if the general results in Ref. [26] for the effective interface potential are applied to the present model one finds the same effective interface potential as the one obtained directly within the present model.

\section{Appendix E: Derivation of the effective interface potential for the model with long-ranged interactions}

The derivation of $\omega(\ell)$ in Eq. (56) follows the same procedure as described in Sec II. We perform a Taylor expansion up to second order of the local part of the functional in Eq. (47) about the sharp-kink profile in Eq. (8) shifted by $d_{w}$ and the sharp-kink profile in Eq. (9) with the bulk state being determined by Eq. (E3) below. From this expansion we obtain an approximate variational functional $\hat{\Omega}_{l r}$ for the model with long-ranged interactions. By subtracting the bulk contribution $\Omega_{b, l r}$ of the gas phase we obtain the surface contribution $\Omega_{s, l r}$ to this variational functional:

$$
\begin{aligned}
& \beta \Omega_{s, l r}\left(\ell,\left[\Delta \phi(z), \Delta \rho_{ \pm}(z)\right]\right)=\frac{\beta\left(\hat{\Omega}_{l r}\left(\ell,\left[\Delta \phi(z), \Delta \rho_{ \pm}(z)\right]\right)-V \Omega_{b, l r}\left(\phi_{g}, 0\right)\right)}{A} \\
& =\beta \ell\left[\Omega_{b, l r}\left(\phi_{l}, I\right)-\Omega_{b, l r}\left(\phi_{g}, 0\right)\right]-\beta d_{w} \Omega_{b, l r}\left(\phi_{l}, I\right) \\
& +\int_{d_{w}}^{\ell} d z\left\{\frac{\chi(T)}{6}\left(\frac{d}{d z} \Delta \phi(z)\right)^{2}+\frac{1}{2}(\Delta \phi(z))^{2}\left(\frac{1}{\phi_{l}}+\frac{1}{1-\phi_{l}}-2 \chi(T)\right)\right\} \\
& +\int_{\ell}^{\infty} d z\left\{\frac{\chi(T)}{6}\left(\frac{d}{d z} \Delta \phi(z)\right)^{2}+\frac{1}{2}(\Delta \phi(z))^{2}\left(\frac{1}{\phi_{g}}+\frac{1}{1-\phi_{g}}-2 \chi(T)\right)\right\} \\
& -\beta h_{1} \phi_{l}-\beta h_{1} \Delta \phi\left(d_{w}\right)+\beta \frac{g}{2}\left(\phi_{l}+\Delta \phi\left(d_{w}\right)\right)^{2}
\end{aligned}
$$



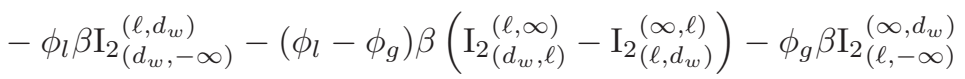

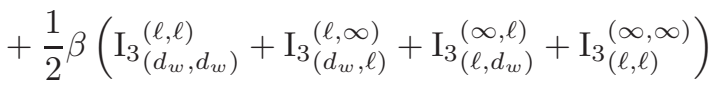

$$
\begin{aligned}
& +\phi_{l} \rho_{w} \beta \int_{d_{w}}^{\ell} d z V(z)+\rho_{w} \beta \mathrm{I}_{1}^{\stackrel{(\ell)}{\left(d_{w}\right)}} \\
& +\phi_{g} \rho_{w} \beta \int_{\ell}^{\infty} d z V(z)+\rho_{w} \beta \mathbf{I}_{1}^{(\ell)} \\
& +\int_{0}^{\ell} d z\left\{\frac{1}{2 I} \sum_{i= \pm}\left(\Delta \rho_{i}(z)\right)^{2}+\frac{2 \pi l_{B}}{\varepsilon_{l}}\left(D\left(z,\left[\Delta \rho_{ \pm}\right]\right)\right)^{2}\right\},
\end{aligned}
$$

where $d_{w}$ describes the excluded volume due to the repulsive part of the substrate potential $V(z)$ given by Eq. (46) and

$$
w\left(\left|z-z^{\prime}\right|\right)=\frac{\pi A_{f}}{2\left[\left(z-z^{\prime}\right)^{2}+1\right]^{2}} .
$$

The bulk grand canonical potential density $\Omega_{b, l r}$ per $k_{B} T$ is given by

$$
\begin{aligned}
\beta \Omega_{b, l r}(\phi, \rho) & =\phi\left(\ln (\phi)-\beta \mu_{\phi}\right)+(1-\phi) \ln (1-\phi)+\chi(T) \phi(1-\phi)+\frac{1}{2} \phi^{2} \int_{-\infty}^{\infty} d x w(|x|) \\
& +2 \rho(\ln (\rho)-1)-\beta \mu_{I} \rho+\rho\left(V_{+}(\phi)+V_{-}(\phi)\right) .
\end{aligned}
$$

$I_{0}, I_{1}, I_{2}$, and $I_{3}$ are abbreviations for the following types of integrals:

$$
\mathrm{I}_{0}^{\left(u_{2}, v_{2}\right)}=\int_{\left.u_{1}, v_{1}\right)}^{u_{2}} d z \int_{v_{1}}^{v_{2}} d z^{\prime} w\left(\left|z-z^{\prime}\right|\right),
$$




$$
\begin{gathered}
\mathrm{I}_{1}^{\left(\begin{array}{c}
\left(u_{2}\right) \\
\left(u_{1}\right)
\end{array}\right)}=\int_{u_{1}}^{u_{2}} d z V(z) \Delta \phi(z), \\
\mathrm{I}_{2}^{\left(\begin{array}{c}
\left(u_{2}, v_{2}\right) \\
\left(u_{1}, v_{1}\right)
\end{array}\right)} \int_{u_{1}}^{u_{2}} d z \int_{v_{1}}^{v_{2}} d z^{\prime} \Delta \phi(z) w\left(\left|z-z^{\prime}\right|\right),
\end{gathered}
$$

and

$$
\mathrm{I}_{3}\left(\begin{array}{l}
\left(u_{2}, v_{2}\right) \\
\left.u_{1}, v_{1}\right)
\end{array}=\int_{u_{1}}^{u_{2}} d z \int_{v_{1}}^{v_{2}} d z^{\prime} \Delta \phi(z) \Delta \phi\left(z^{\prime}\right) w\left(\left|z-z^{\prime}\right|\right) .\right.
$$

The integrals in Eqs. (E5)-(E7) are evaluated at two-phase coexistence using the solutions for $\Delta \phi(z)$ obtained in Sec. II (see Eqs. (22) and (29)). We are interested in the asymptotic behavior of these integrals in the limit $\ell \rightarrow \infty$. Using Eqs. (22) and (46), $\mathrm{I}_{1}^{(\ell)}\left(d_{w}\right)$ can be written as

$$
\begin{aligned}
\mathrm{I}_{1}^{(\ell)}\left(d_{w}\right) & =-\int_{d_{w}}^{\ell} d z\left[\sum_{i \geq 3} \frac{u_{i}}{z^{i}}\right]\left[A_{l} \exp (z / \xi)+B_{l} \exp (-z / \xi)\right] \\
& =-A_{l} \int_{d_{w}}^{\ell} d z\left[\sum_{i \geq 3} \frac{u_{i}}{z^{i}}\right] \exp (z / \xi)-B_{l} \int_{d_{w}}^{\ell} d z\left[\sum_{i \geq 3} \frac{u_{i}}{z^{i}}\right] \exp (-z / \xi) .
\end{aligned}
$$

Asymptotic approximations for the integrals in Eq. (E8) are obtained via integrating by parts repeatedly:

$$
\begin{aligned}
\int_{d_{w}}^{\ell} d z \frac{\exp (z / \xi)}{z^{3}}= & \frac{\xi \exp (\ell / \xi)}{\ell^{3}}-\frac{\xi \exp \left(d_{w} / \xi\right)}{d_{w}^{3}} \\
& +3 \xi \int_{d_{w}}^{\ell} d z \frac{\exp (z / \xi)}{z^{4}} \\
& =\frac{\xi \exp (\ell / \xi)}{\ell^{3}}+\mathcal{O}\left(\ell^{-4} \exp (\ell / \xi)\right), \ell \gg d_{w}
\end{aligned}
$$

and

$$
\int_{d_{w}}^{\ell} d z \frac{\exp (-z / \xi)}{z^{3}}=\int_{d_{w}}^{\infty} d z \frac{\exp (-z / \xi)}{z^{3}}-\int_{\ell}^{\infty} d z \frac{\exp (-z / \xi)}{z^{3}}
$$

with

$$
\begin{aligned}
\int_{\ell}^{\infty} d z \frac{\exp (-z / \xi)}{z^{3}}= & \frac{\xi \exp (-\ell / \xi)}{\ell^{3}}-3 \xi \int_{\ell}^{\infty} d z \frac{\exp (-z / \xi)}{z^{4}} \\
= & \frac{\xi \exp (-\ell / \xi)}{\ell^{3}}+\mathcal{O}\left(\ell^{-4} \exp (\ell / \xi)\right) \\
& \ell \gg d_{w}
\end{aligned}
$$

Here and in the following we have used the properties

$$
\int_{a}^{\ell+b} d z \frac{P_{1}(z)}{P_{2}(z)} \exp (z / \xi) \stackrel{\ell \rightarrow \infty}{\simeq} \frac{c_{1}}{c_{2}} \xi \ell^{n_{1}-n_{2}} \exp (\ell / \xi)
$$

and

$$
\int_{\ell+a}^{\infty} d z \frac{P_{1}(z)}{P_{2}(z)} \exp (-z / \xi) \stackrel{\ell \rightarrow \infty}{\simeq} \frac{c_{1}}{c_{2}} \xi \ell^{n_{1}-n_{2}} \exp (-\ell / \xi)
$$

for two polynomials $P_{1}(z)$ and $P_{2}(z)$ of degrees $n_{1}$ and $n_{2}$ with the leading coefficients $c_{1}$ and $c_{2}$, respectively, of the leading terms which follows from L'Hôpital's rule. 
For convenience, we write the expressions in Eq. (29) as

$$
\begin{aligned}
& A_{l}=A_{1} \exp (-\ell / \xi) \\
& B_{l}=B_{1} \exp (-\ell / \xi)+B_{2}, \\
& B_{g}=-A_{1} \exp (\ell / \xi)+B_{1} \exp (-\ell / \xi)+B_{2},
\end{aligned}
$$

with

$$
\begin{aligned}
A_{1} & =\frac{\phi_{g}-\phi_{l}}{2}, \\
B_{1} & =\frac{\left(\frac{\chi(T)}{3 \xi}-\beta g\right)\left(\phi_{g}-\phi_{l}\right)}{2\left(\beta g+\frac{\chi(T)}{3 \xi}\right)}, \\
B_{2} & =\frac{\beta\left(h_{1}-g \phi_{l}\right)}{\beta g+\frac{\chi(T)}{3 \xi}} .
\end{aligned}
$$

Collecting only algebraic terms up to the order $1 / \ell^{3}$, one obtains for $\mathrm{I}_{1}^{(\ell)}\left(d_{w}\right)$

$$
\mathrm{I}_{1}^{(\ell)}\left(d_{w}\right)=-\frac{\xi A_{1} u_{3}}{\ell^{3}}+\mathcal{O}\left(\frac{1}{\ell^{4}}\right) .
$$

Analogously, for $\mathrm{I}_{1}^{(\infty)}(\ell)$ one has

$$
\begin{aligned}
\mathrm{I}_{1}^{(\ell)} & =-\int_{\ell}^{\infty} d z\left[\sum_{i \geq 3} \frac{u_{i}}{z^{i}}\right] B_{g} \exp (-z / \xi) \\
& =\frac{\xi A_{1} u_{3}}{\ell^{3}}+\mathcal{O}\left(\frac{1}{\ell^{4}}\right)
\end{aligned}
$$

In order to calculate integrals of the type $I_{2}$ we first integrate Eq. (E2) using the various integration limits appearing in Eq. (E1) so that

$$
\begin{gathered}
\int_{-\infty}^{d_{w}} d z^{\prime} w\left(\left|z-z^{\prime}\right|\right)=\frac{\pi A_{f}}{4}\left[\frac{\pi}{2}-\arctan \left(z-d_{w}\right)-\frac{z-d_{w}}{\left(z-d_{w}\right)^{2}+1}\right] \\
\int_{\ell}^{\infty} d z^{\prime} w\left(\left|z-z^{\prime}\right|\right)=\frac{\pi A_{f}}{4}\left[\arctan (z-\ell)+\frac{z-\ell}{(z-\ell)^{2}+1}+\frac{\pi}{2}\right]
\end{gathered}
$$

and

$$
\int_{d_{w}}^{\ell} d z^{\prime} w\left(\left|z-z^{\prime}\right|\right)=\frac{\pi A_{f}}{4}\left[\arctan \left(z-d_{w}\right)+\frac{z-d_{w}}{\left(z-d_{w}\right)^{2}+1}-\arctan (z-\ell)-\frac{z-\ell}{(z-\ell)^{2}+1}\right] .
$$

Using Eqs. (22) and (E16) we can write $\mathrm{I}_{2}^{\left(\ell, d_{w}\right)}\left(d_{w},-\infty\right)$ as

$$
\begin{aligned}
\left.\mathrm{I}_{2}^{\left(\ell, d_{w}\right)},-\infty\right) & =\frac{\pi A_{f}}{4} \int_{d_{w}}^{\ell} d z\left[\frac{\pi}{2}-\arctan \left(z-d_{w}\right)-\frac{z-d_{w}}{\left(z-d_{w}\right)^{2}+1}\right]\left[A_{l} \exp (z / \xi)+B_{l} \exp (-z / \xi)\right] \\
& =\frac{\pi A_{f}}{4} A_{l}\left[\frac{\xi \pi}{2}\left(\exp (\ell / \xi)-\exp \left(d_{w} / \xi\right)\right)-\xi \exp (\ell / \xi) \arctan \left(\ell-d_{w}\right)\right. \\
& \left.+\xi \exp \left(d_{w} / \xi\right) \int_{0}^{\ell-d_{w}} d y \frac{\exp (y / \xi)}{y^{2}+1}-\exp \left(d_{w} / \xi\right) \int_{0}^{\ell-d_{w}} d z \frac{y \exp (y / \xi)}{y^{2}+1}\right] \\
& +\frac{\pi A_{f}}{4} B_{l}\left[\frac{-\xi \pi}{2}\left(\exp (-\ell / \xi)-\exp \left(-d_{w} / \xi\right)\right)+\xi \exp (-\ell / \xi) \arctan \left(\ell-d_{w}\right)\right. \\
& \left.-\xi \exp \left(-d_{w} / \xi\right) \int_{0}^{\ell-d_{w}} d y \frac{\exp (-y / \xi)}{y^{2}+1}-\exp \left(-d_{w} / \xi\right) \int_{0}^{\ell-d_{w}} d y \frac{y \exp (-y / \xi)}{y^{2}+1}\right]
\end{aligned}
$$


where we have changed the integration variable to $y=z-d_{w}$. Asymptotic approximations for the integrals in Eq. (E19) are obtained via integrating by parts repeatedly:

$$
\begin{gathered}
\int_{0}^{\ell-d_{w}} d z \frac{\exp (z / \xi)}{z^{2}+1}=\frac{\xi \exp \left(\left(\ell-d_{w}\right) / \xi\right)}{\left(\ell-d_{w}\right)^{2}+1}-\xi+\frac{2\left(\ell-d_{w}\right) \xi^{2} \exp \left(\left(\ell-d_{w}\right) / \xi\right)}{\left(\left(\ell-d_{w}\right)^{2}+1\right)^{2}} \\
+\xi^{2} \int_{0}^{\ell-d_{w}} d z \frac{6 z^{2}-2}{\left(z^{2}+1\right)^{3}} \exp (z / \xi), \\
=\frac{\xi \exp \left(\left(\ell-d_{w}\right) / \xi\right)}{\ell^{2}}+\frac{2\left(d_{w} \xi+\xi^{2}\right) \exp \left(\left(\ell-d_{w}\right) / \xi\right)}{\ell^{3}} \\
+\mathcal{O}\left(\ell^{-4} \exp (\ell / \xi)\right), \quad \ell \gg d_{w}, \\
\int_{0}^{\ell-d_{w}} d z \frac{z \exp (z / \xi)}{z^{2}+1}= \\
+\frac{\xi\left(\ell-d_{w}\right) \exp \left(\left(\ell-d_{w}\right) / \xi\right)}{\left(\ell-d_{w}\right)^{2}+1}-\frac{\xi^{2} \exp \left(\left(\ell-d_{w}\right) / \xi\right)}{\left(\ell-d_{w}\right)^{2}+1}+\xi^{2} \\
\left(\left(\ell-d_{w}\right)^{2}+1\right)^{2} \\
+\frac{8\left(\left(\ell-d_{w}\right) \xi\right)^{3} \exp \left(\left(\ell-d_{w}\right) / \xi\right)}{\left(\left(\ell-d_{w}\right)^{2}+1\right)^{3}}+\int_{0}^{\ell-d_{w}} d z \frac{6\left(z^{4}-6 z^{2}+1\right)}{\left(z^{2}+1\right)^{4}} \xi^{3} \exp (z / \xi), \\
= \\
\xi \exp \left(\left(\ell-d_{w}\right) / \xi\right)\left(\frac{1}{\ell}+\frac{d_{w}}{\ell^{2}}+\frac{d_{w}^{2}-1}{\ell^{3}}\right)+\frac{\xi^{2} \exp \left(\left(\ell-d_{w}\right) / \xi\right)}{\ell^{2}} \\
+\frac{2 \xi^{2}\left(d_{w}+\xi\right) \exp \left(\left(\ell-d_{w}\right) / \xi\right)}{\ell^{3}}+\mathcal{O}\left(\ell^{-4} \exp (\ell / \xi)\right), \quad \ell \gg d_{w},
\end{gathered}
$$

and

$$
\int_{0}^{\ell-d_{w}} d z \frac{\exp (-z / \xi)}{z^{2}+1}=\int_{0}^{\infty} d z \frac{\exp (-z / \xi)}{z^{2}+1}-\int_{\ell-d_{w}}^{\infty} d z \frac{\exp (-z / \xi)}{z^{2}+1}
$$

with

$$
\begin{aligned}
\int_{\ell-d_{w}}^{\infty} d z \frac{\exp (-z / \xi)}{z^{2}+1} & =\frac{\xi \exp \left(-\left(\ell-d_{w}\right) / \xi\right)}{\left(\ell-d_{w}\right)^{2}+1}-\frac{2\left(\ell-d_{w}\right) \xi^{2} \exp \left(-\left(\ell-d_{w}\right) / \xi\right)}{\left(\left(\ell-d_{w}\right)^{2}+1\right)^{2}} \\
& +\int_{\ell-d_{w}}^{\infty} d z \frac{6 z^{2}-2}{\left(z^{2}+1\right)^{3}} \xi^{2} \exp (-z / \xi) \\
& =\frac{\xi \exp \left(-\left(\ell-d_{w}\right) / \xi\right)}{\ell^{2}}+\frac{2 \xi\left(d_{w}-\xi\right) \exp \left(-\left(\ell-d_{w}\right) / \xi\right)}{\ell^{3}} \\
& +\mathcal{O}\left(\ell^{-4} \exp (-\ell / \xi)\right), \quad \ell \gg d_{w},
\end{aligned}
$$

and

$$
\int_{0}^{\ell-d_{w}} d z \frac{z \exp (-z / \xi)}{z^{2}+1}=\int_{0}^{\infty} d z \frac{z \exp (-z / \xi)}{z^{2}+1}-\int_{\ell-d_{w}}^{\infty} d z \frac{z \exp (-z / \xi)}{z^{2}+1}
$$

with

$$
\begin{aligned}
\int_{\ell-d_{w}}^{\infty} d z \frac{z \exp (-z / \xi)}{z^{2}+1} & =\frac{\xi\left(\ell-d_{w}\right) \exp \left(-\left(\ell-d_{w}\right) / \xi\right)}{\left(\ell-d_{w}\right)^{2}+1}+\frac{\xi^{2} \exp \left(-\left(\ell-d_{w}\right) / \xi\right)}{\ell^{2}+1} \\
& -\frac{2\left(\left(\ell-d_{w}\right) \xi\right)^{2} \exp \left(-\left(\ell-d_{w}\right) / \xi\right)}{\left(\left(\ell-d_{w}\right)^{2}+1\right)^{2}}-\frac{6\left(\ell-d_{w}\right) \xi^{3} \exp \left(-\left(\ell-d_{w}\right) / \xi\right)}{\left(\left(\ell-d_{w}\right)^{2}+1\right)^{2}} \\
& +\frac{8\left(\left(\ell-d_{w}\right) \xi\right)^{3} \exp \left(-\left(\ell-d_{w}\right) / \xi\right)}{\left(\left(\ell-d_{w}\right)^{2}+1\right)^{3}}-\xi^{3} \int_{\ell-d_{w}}^{\infty} d z \frac{6\left(z^{4}-6 z^{2}+1\right)}{\left(^{2}+1\right)^{4}} \exp (-z / \xi), \\
& =\xi \exp \left(-\left(\ell-d_{w}\right) / \xi\right)\left(\frac{1}{\ell}+\frac{d_{w}}{\ell^{2}}+\frac{d_{w}^{2}-1}{\ell^{3}}\right)-\frac{\xi^{2} \exp \left(-\left(\ell-d_{w}\right) / \xi\right)}{\ell^{2}} \\
& +\frac{2 \xi^{2}\left(\xi-d_{w}\right) \exp \left(-\left(\ell-d_{w}\right) / \xi\right)}{\ell^{3}}+\mathcal{O}\left(\ell^{-4} \exp (-\ell / \xi)\right), \quad \ell \gg d_{w} .
\end{aligned}
$$


Additionally, for $\ell \gg d_{w}$ one has

$$
\frac{\pi}{2}-\arctan \left(\ell-d_{w}\right)=\frac{1}{\ell}+\frac{d_{w}}{\ell^{2}}+\frac{d_{w}^{2}-1 / 3}{\ell^{3}}+\mathcal{O}\left(\frac{1}{\ell^{4}}\right) .
$$

Note that Eqs. (E20) and (E21) contain terms which increase exponentially with $\ell$. However, these two integrals are multiplied by $A_{l}$ which decays exponentially with $\ell$ (see Eqs. (E8) and (E12)).

Collecting constants and algebraic terms up to the order $1 / \ell^{3}$, for $\mathrm{I}_{2}^{\left(\ell, d_{w}\right)}\left(d_{w},-\infty\right)$ one obtains

$$
\begin{aligned}
\left.\mathrm{I}_{2}^{\left(\ell, d_{w}\right)},-\infty\right) & =\frac{A_{f} \pi B_{2}}{4} \exp \left(-d_{w} / \xi\right)\left(\frac{\xi \pi}{2}-\xi \int_{0}^{\infty} d y \frac{\exp \left(-\frac{y}{\xi}\right)}{y^{2}+1}-\int_{0}^{\infty} d y \frac{y \exp \left(-\frac{y}{\xi}\right)}{y^{2}+1}\right) \\
& +\frac{\pi A_{f} \xi A_{1}}{6 \ell^{3}}+\mathcal{O}\left(\frac{1}{\ell^{4}}\right) .
\end{aligned}
$$

Similarly, $\mathrm{I}_{2}^{(}(\ell,-\infty)$ can be written as

$$
\begin{aligned}
\mathrm{I}_{2}^{(\ell,-\infty)}\left(d_{w}\right) & =\frac{\pi A_{f}}{4} \int_{\ell}^{\infty} d z\left[\frac{\pi}{2}-\arctan \left(z-d_{w}\right)-\frac{z-d_{w}}{\left(z-d_{w}\right)^{2}+1}\right] B_{g} \exp (-z / \xi) \\
& =-\frac{\pi A_{f} \xi A_{1}}{6 \ell^{3}}+\mathcal{O}\left(\frac{1}{\ell^{4}}\right) .
\end{aligned}
$$

Using Eqs. (22) and (E17) we can write $\mathrm{I}_{2}^{(\ell, \infty)}\left(d_{w}, \ell\right)$ as

$$
\begin{aligned}
\mathrm{I}_{2}^{(\ell, \infty)}\left(d_{w}, \ell\right) & =\frac{\pi A_{f}}{4} \int_{d_{w}}^{\ell} d z\left[\arctan (z-\ell)+\frac{z-\ell}{(z-\ell)^{2}+1}+\frac{\pi}{2}\right]\left[A_{l} \exp (z / \xi)+B_{l} \exp (-z / \xi)\right] \\
& =\frac{\pi A_{f}}{4} A_{l}\left[\frac{\xi \pi}{2}\left(\exp (\ell / \xi)-\exp \left(d_{w} / \xi\right)\right)+\xi \exp \left(d_{w} / \xi\right) \arctan \left(\ell-d_{w}\right)\right. \\
& \left.-\xi \exp (\ell / \xi) \int_{0}^{\ell-d_{w}} d y \frac{\exp (-y / \xi)}{y^{2}+1}-\exp (\ell / \xi) \int_{0}^{\ell-d_{w}} d z \frac{y \exp (-y / \xi)}{y^{2}+1}\right] \\
& +\frac{\pi A_{f}}{4} B_{l}\left[\frac{-\xi \pi}{2}\left(\exp (-\ell / \xi)-\exp \left(-d_{w} / \xi\right)\right)-\xi \exp \left(-d_{w} / \xi\right) \arctan \left(\ell-d_{w}\right)\right. \\
& \left.+\xi \exp (-\ell / \xi) \int_{0}^{\ell-d_{w}} d y \frac{\exp (y / \xi)}{y^{2}+1}-\exp (-\ell / \xi) \int_{0}^{\ell-d_{w}} d y \frac{y \exp (y / \xi)}{y^{2}+1}\right]
\end{aligned}
$$

where we have changed the integration variable to $y=\ell-z$. Using Eqs. (E20)-(E26) one obtains asymptotically

$$
\begin{aligned}
\mathrm{I}_{2}^{(\ell, \infty)}\left(d_{w}, \ell\right) & =\frac{A_{f} \pi A_{1}}{4}\left(\frac{\xi \pi}{2}-\xi \int_{0}^{\infty} d y \frac{\exp \left(-\frac{y}{\xi}\right)}{y^{2}+1}-\int_{0}^{\infty} d y \frac{y \exp \left(-\frac{y}{\xi}\right)}{y^{2}+1}\right) \\
& +\frac{A_{f} \xi \pi B_{2}}{6 \ell^{3}} \exp \left(-d_{w} / \xi\right)+\mathcal{O}\left(\frac{1}{\ell^{4}}\right) .
\end{aligned}
$$

Similarly, $\mathrm{I}_{2}^{\left(\ell, d_{w}\right)}$ can be written as (see Eqs. (22) and (E18)

$$
\begin{aligned}
\mathrm{I}_{2}^{(\ell, \ell)}\left(\begin{array}{c}
(\infty, \ell) \\
(\ell)
\end{array}\right. & \int_{\ell}^{\infty} d z \frac{\pi A_{f}}{4}\left[\arctan \left(z-d_{w}\right)+\frac{z-d_{w}}{\left(z-d_{w}\right)^{2}+1}-\arctan (z-\ell)-\frac{z-\ell}{(z-\ell)^{2}+1}\right] \times \\
& \times\left[B_{l}-A_{l} \exp (2 \ell / \xi)\right] \exp (-z / \xi) \\
& =\frac{\pi A_{f}}{4}\left[B_{l}-A_{l} \exp (2 \ell / \xi)\right]\left[\xi \arctan \left(\ell-d_{w}\right) \exp (-\ell / \xi)\right. \\
& +\xi \exp \left(-d_{w} / \xi\right) \int_{\ell-d_{w}}^{\infty} d y \frac{\exp (-y / \xi)}{y^{2}+1}+\exp \left(-d_{w} / \xi\right) \int_{\ell-d_{w}}^{\infty} d y \frac{y \exp (-y / \xi)}{y^{2}+1} \\
& \left.-\xi \exp (-\ell / \xi) \int_{0}^{\infty} d y^{\prime} \frac{\exp \left(-y^{\prime} / \xi\right)}{y^{\prime 2}+1}-\exp (-\ell / \xi) \int_{0}^{\infty} d y^{\prime} \frac{y^{\prime} \exp \left(-y^{\prime} / \xi\right)}{y^{\prime 2}+1}\right],
\end{aligned}
$$


where we have changed the integration variable to $y=z-d_{w}$ and $y^{\prime}=z-\ell$, respectively. Using Eqs. (E20)-(E26), this leads to the asymptotic behavior

$$
\begin{aligned}
\mathrm{I}_{2}^{(\ell, \ell)}(\ell) & =-\frac{A_{f} \pi A_{1}}{4}\left(\frac{\xi \pi}{2}-\xi \int_{0}^{\infty} d y \frac{\exp \left(-\frac{y}{\xi}\right)}{y^{2}+1}-\int_{0}^{\infty} d y \frac{y \exp \left(-\frac{y}{\xi}\right)}{y^{2}+1}\right) \\
& +\frac{\pi A_{f} \xi A_{1}}{6 \ell^{3}}+\mathcal{O}\left(\frac{1}{\ell^{4}}\right) .
\end{aligned}
$$

Integrals of the type $\mathrm{I}_{3}$ can be written as (see Eqs. (22) and Eqs. (E2))

$$
\begin{aligned}
& \mathrm{I}_{3}^{(\ell, \ell)}{ }_{\left(d_{w}, d_{w}\right)}^{(\ell)}=\frac{A_{f} \pi}{2}\left\{A_{l}^{2} \int_{d_{w}}^{\ell} d z \int_{d_{w}}^{\ell} d z^{\prime} \frac{\exp (z / \xi) \exp \left(z^{\prime} / \xi\right)}{\left[\left(z-z^{\prime}\right)^{2}+1\right]^{2}}\right. \\
& +2 A_{l} B_{l} \int_{d_{w}}^{\ell} d z \int_{d_{w}}^{\ell} d z^{\prime} \frac{\exp (z / \xi) \exp \left(-z^{\prime} / \xi\right)}{\left[\left(z-z^{\prime}\right)^{2}+1\right]^{2}} \\
& \left.+B_{l}^{2} \int_{d_{w}}^{\ell} d z \int_{d_{w}}^{\ell} d z^{\prime} \frac{\exp (-z / \xi) \exp \left(-z^{\prime} / \xi\right)}{\left[\left(z-z^{\prime}\right)^{2}+1\right]^{2}}\right\}, \\
& \mathrm{I}_{3(\ell, \ell)}^{(\infty, \infty)}=\frac{A_{f} \pi}{2} B_{g}^{2} \int_{\ell}^{\infty} d z \int_{\ell}^{\infty} d z^{\prime} \frac{\exp (-z / \xi) \exp \left(-z^{\prime} / \xi\right)}{\left[\left(z-z^{\prime}\right)^{2}+1\right]^{2}} \\
& \mathrm{I}_{3}^{(\ell, \infty)}\left(d_{w}, \ell\right)=\frac{A_{f} \pi}{2}\left\{A_{l} B_{g} \int_{d_{w}}^{\ell} d z \int_{\ell}^{\infty} d z^{\prime} \frac{\exp (z / \xi) \exp \left(-z^{\prime} / \xi\right)}{\left[\left(z-z^{\prime}\right)^{2}+1\right]^{2}}\right. \\
& \left.+B_{l} B_{g} \int_{d_{w}}^{\ell} d z \int_{\ell}^{\infty} d z^{\prime} \frac{\exp (-z / \xi) \exp \left(-z^{\prime} / \xi\right)}{\left[\left(z-z^{\prime}\right)^{2}+1\right]^{2}}\right\},
\end{aligned}
$$

and

$$
\begin{aligned}
\mathrm{I}_{3}\left(\ell, d_{w}\right) & =\frac{A_{f} \pi}{2}\left\{A_{l} B_{g} \int_{\ell}^{\infty} d z \int_{d_{w}}^{\ell} d z^{\prime} \frac{\exp (-z / \xi) \exp \left(z^{\prime} / \xi\right)}{\left[\left(z-z^{\prime}\right)^{2}+1\right]^{2}}\right. \\
& \left.+B_{l} B_{g} \int_{\ell}^{\infty} d z \int_{d_{w}}^{\ell} d z^{\prime} \frac{\exp (-z / \xi) \exp \left(-z^{\prime} / \xi\right)}{\left[\left(z-z^{\prime}\right)^{2}+1\right]^{2}}\right\},
\end{aligned}
$$

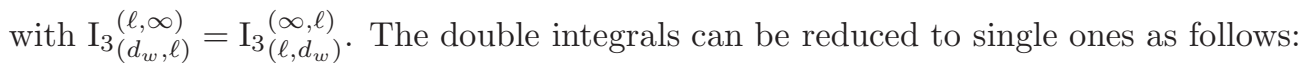

$$
\begin{aligned}
& \int_{u_{1}}^{u_{2}} d z \int_{v_{1}}^{v_{2}} d z^{\prime} \frac{\exp (z / \xi) \exp \left(z^{\prime} / \xi\right)}{\left[\left(z-z^{\prime}\right)^{2}+1\right]^{2}}=\int_{u_{1}}^{u_{2}} d z \exp (2 z / \xi) \int_{v_{1}}^{v_{2}} d z^{\prime} \frac{\exp \left(\left(z^{\prime}-z\right) / \xi\right)}{\left[\left(z-z^{\prime}\right)^{2}+1\right]^{2}} \\
& \stackrel{\left(y:=z^{\prime}-z\right)}{=} \int_{u_{1}}^{u_{2}} d z \exp (2 z / \xi) \int_{v_{1}-z}^{v_{2}-z} d y \frac{\exp (y / \xi)}{\left[y^{2}+1\right]^{2}} \\
&=\left.\frac{\xi}{2}\left[\exp (2 z / \xi) \int_{v_{1}-z}^{v_{2}-z} d y \frac{\exp (y / \xi)}{\left[y^{2}+1\right]^{2}}\right]\right|_{z=u_{1}} ^{z=u_{2}} \\
&-\frac{\xi}{2} \int_{u_{1}}^{u_{2}} d z \exp (2 z / \xi)\left[-\frac{\exp \left(\left(v_{2}-z\right) / \xi\right)}{\left[\left(v_{2}-z\right)^{2}+1\right]^{2}}\right. \\
&\left.+\frac{\exp \left(\left(v_{1}-z\right) / \xi\right)}{\left[\left(v_{1}-z\right)^{2}+1\right]^{2}}\right] \\
&(y\left.:=z-v_{2,1}\right)=\left.\frac{\xi}{2}\left[\exp (2 z / \xi) \int_{v_{1}-z}^{v_{2}-z} d y \frac{\exp (y / \xi)}{\left[y^{2}+1\right]^{2}}\right]\right|_{z=u_{1}} ^{z=u_{2}} \\
&+\left.\frac{\xi}{2}\left[\exp (2 z / \xi) \int_{u_{1}-z}^{u_{2}-z} d y \frac{\exp (y / \xi)}{\left(y^{2}+1\right)^{2}}\right]\right|_{z=v_{1}} ^{z=v_{2}}
\end{aligned}
$$




$$
\begin{aligned}
\int_{u_{1}}^{u_{2}} d z \int_{v_{1}}^{v_{2}} d z^{\prime} \frac{\exp (-z / \xi) \exp \left(-z^{\prime} / \xi\right)}{\left[\left(z-z^{\prime}\right)^{2}+1\right]^{2}} & =-\left.\frac{\xi}{2}\left[\exp (-2 z / \xi) \int_{v_{1}-z}^{v_{2}-z} d y \frac{\exp (-y / \xi)}{\left(y^{2}+1\right)^{2}}\right]\right|_{z=u_{1}} ^{z=u_{2}} \\
& -\left.\frac{\xi}{2}\left[\exp (-2 z / \xi) \int_{u_{1}-z}^{u_{2}-z} d y \frac{\exp (-y / \xi)}{\left(y^{2}+1\right)^{2}}\right]\right|_{z=v_{1}} ^{z=v_{2}}
\end{aligned}
$$

and

$$
\begin{aligned}
\int_{u_{1}}^{u_{2}} d z \int_{v_{1}}^{v_{2}} d z^{\prime} \frac{\exp (z / \xi) \exp \left(-z^{\prime} / \xi\right)}{\left[\left(z-z^{\prime}\right)^{2}+1\right]^{2}} & =\left.\left[z \int_{v_{1}-z}^{v_{2}-z} d y \frac{\exp (-y / \xi)}{\left[y^{2}+1\right]^{2}}\right]\right|_{z=u_{1}} ^{z=u_{2}} \\
+ & {\left.\left[\int_{u_{1}-z}^{u_{2}-z} d y(y+z) \frac{\exp (y / \xi)}{\left[y^{2}+1\right]^{2}}\right]\right|_{z=v_{1}} ^{z=v_{2}} } \\
& =\left.\left[z \int_{v_{1}-z}^{v_{2}-z} d y \frac{\exp (-y / \xi)}{\left[y^{2}+1\right]^{2}}\right]\right|_{z=u_{1}} ^{z=u_{2}} \\
+ & {\left.\left[z \int_{u_{1}-z}^{u_{2}-z} d y \frac{\exp (y / \xi)}{\left[y^{2}+1\right]^{2}}\right]\right|_{z=v_{1}} ^{z=v_{2}} } \\
+ & {\left.\left[\int_{u_{1}-z}^{u_{2}-z} d y \frac{y \exp (y / \xi)}{\left[y^{2}+1\right]^{2}}\right]\right|_{z=v_{1}} ^{z=v_{2}} }
\end{aligned}
$$

Inserting these expressions into Eqs. E33)-E35 leads to

$$
\begin{aligned}
& \mathrm{I}_{3}^{(\ell, \ell)}\left(d_{w}, d_{w}\right)=\frac{A_{f} \pi}{2}\left\{A_{l}^{2} \xi\left[\exp (2 \ell / \xi) \int_{0}^{\ell-d_{w}} d y \frac{\exp (-y / \xi)}{\left(y^{2}+1\right)^{2}}-\exp \left(2 d_{w} / \xi\right) \int_{0}^{\ell-d_{w}} d y \frac{\exp (y / \xi)}{\left(y^{2}+1\right)^{2}}\right]\right. \\
&+2 A_{l} B_{l}\left[\left(\ell-d_{w}\right)\left(\int_{0}^{\ell-d_{w}} d y \frac{\exp (-y / \xi)}{\left(y^{2}+1\right)^{2}}+\int_{0}^{\ell-d_{w}} d y \frac{\exp (y / \xi)}{\left(y^{2}+1\right)^{2}}\right)\right. \\
&\left.-\int_{0}^{\ell-d_{w}} d y \frac{y \exp (-y / \xi)}{\left(y^{2}+1\right)^{2}}-\int_{0}^{\ell-d_{w}} d y \frac{y \exp (y / \xi)}{\left(y^{2}+1\right)^{2}}\right] \\
&+ B_{l}^{2} \xi\left[-\exp (-2 \ell / \xi) \int_{0}^{\ell-d_{w}} d y \frac{\exp (y / \xi)}{\left.\left.\left(y^{2}+1\right)^{2}+\exp \left(-2 d_{w} / \xi\right) \int_{0}^{\ell-d_{w}} d y \frac{\exp (-y / \xi)}{\left(y^{2}+1\right)^{2}}\right]\right\}}\right. \\
& \mathrm{I}_{3}^{(\infty, \ell)}=\frac{A_{f} \pi}{2} B_{g}^{2} \xi \exp (-2 \ell / \xi) \int_{0}^{\infty} d y \frac{\exp (-y / \xi)}{\left(y^{2}+1\right)^{2}}
\end{aligned}
$$

and

$$
\begin{aligned}
\mathrm{I}_{3}^{(\ell, \infty)}\left(d_{w}, \ell\right) & =\frac{A_{f} \pi}{2}\left\{A _ { l } B _ { g } \left[\ell\left(\int_{0}^{\infty} d y \frac{\exp (-y / \xi)}{\left(y^{2}+1\right)^{2}}-\int_{0}^{\ell-d_{w}} d y \frac{\exp (-y / \xi)}{\left(y^{2}+1\right)^{2}}\right)+\int_{0}^{\ell-d_{w}} d y \frac{y \exp (-y / \xi)}{\left(y^{2}+1\right)^{2}}\right.\right. \\
& \left.-d_{w} \int_{l-d_{w}}^{\infty} d y \frac{\exp (-y / \xi)}{\left(y^{2}+1\right)^{2}}\right]+B_{l} B_{g} \frac{\xi}{2}\left[\exp \left(-2 d_{w} / \xi\right) \int_{\ell-d_{w}}^{\infty} d y \frac{\exp (-y / \xi)}{\left(y^{2}+1\right)^{2}}\right. \\
& \left.\left.+\exp (-2 \ell / \xi)\left(\int_{0}^{\ell-d_{w}} d y \frac{\exp (y / \xi)}{\left(y^{2}+1\right)^{2}}-\int_{0}^{\infty} d y \frac{\exp (-y / \xi)}{\left(y^{2}+1\right)^{2}}\right)\right]\right\}
\end{aligned}
$$


We note the following relations:

$$
\begin{aligned}
A_{l}^{2} & =A_{1}^{2} \exp (-2 \ell / \xi) \\
A_{l} B_{l} & =A_{1} B_{1} \exp (-2 \ell / \xi)+A_{1} B_{2} \exp (-\ell / \xi) \\
A_{l} B_{g} & =A_{1} B_{1} \exp (-2 \ell / \xi)+A_{1} B_{2} \exp (-\ell / \xi)-A_{1}^{2} \\
B_{l}^{2} & =B_{1}^{2} \exp (-2 \ell / \xi)+2 B_{1} B_{2} \exp (-\ell / \xi)+B_{2}^{2} \\
B_{l} B_{g} & =B_{1}^{2} \exp (-2 \ell / \xi)+2 B_{1} B_{2} \exp (-\ell / \xi)-A_{1} B_{2} \exp (\ell / \xi)+B_{2}^{2}-A_{1} B_{1} \\
B_{g}^{2} & =B_{1}^{2} \exp (-2 \ell / \xi)+2 B_{1} B_{2} \exp (-\ell / \xi)-2 A_{1} B_{2} \exp (\ell / \xi) \\
& +A_{1}^{2} \exp (2 \ell / \xi)+B_{2}^{2}-2 A_{1} B_{1} .
\end{aligned}
$$

Accordingly we obtain

$$
\begin{aligned}
& \frac{1}{2}\left(\mathrm{I}_{3}^{(\ell, \ell)}{\left(d_{w}, d_{w}\right)}^{(\ell)}+\mathrm{I}_{3}^{(\ell, \infty)}+\mathrm{I}_{3}^{(\infty, \ell)}{ }_{\left(\ell, d_{w}\right)}^{(\infty, \ell)}+\mathrm{I}_{3}^{(\ell, \ell)}\right)=\frac{A_{f} \pi}{2}\left\{\frac { A _ { l } ^ { 2 } \xi } { 2 } \left[\exp (2 \ell / \xi) \int_{0}^{\ell-d_{w}} d y \frac{\exp (-y / \xi)}{\left(y^{2}+1\right)^{2}}\right.\right. \\
& \left.-\exp \left(2 d_{w} / \xi\right) \int_{0}^{\ell-d_{w}} d y \frac{\exp (y / \xi)}{\left(y^{2}+1\right)^{2}}\right] \\
& -A_{1}^{2}\left[\left(\ell-d_{w}\right) \int_{\ell-d_{w}}^{\infty} d y \frac{\exp (-y / \xi)}{\left(y^{2}+1\right)^{2}}+\int_{0}^{\ell-d_{w}} d y \frac{y \exp (-y / \xi)}{\left(y^{2}+1\right)^{2}}\right] \\
& +A_{l} B_{l}\left[\left(\ell-d_{w}\right) \int_{d_{w}-\ell}^{\infty} d y \frac{\exp (-y / \xi)}{\left(y^{2}+1\right)^{2}}-\int_{0}^{\ell-d_{w}} d y \frac{y \exp (y / \xi)}{\left(y^{2}+1\right)^{2}}\right] \\
& +\frac{B_{l}^{2} \xi}{2} \exp \left(-2 d_{w} / \xi\right) \int_{0}^{\infty} d y \frac{\exp (-y / \xi)}{\left(y^{2}+1\right)^{2}} \\
& +\left(-A_{1} B_{1}-A_{1} B_{2} \exp (\ell / \xi)\right) \frac{\xi}{2} \times \\
& \times\left[\exp \left(-2 d_{w} / \xi\right) \int_{\ell-d_{w}}^{\infty} d y \frac{\exp (-y / \xi)}{\left(y^{2}+1\right)^{2}}\right. \\
& \left.+\exp (-2 \ell / \xi) \int_{0}^{\ell-d_{w}} d y \frac{\exp (y / \xi)}{\left(y^{2}+1\right)^{2}}\right] \\
& +\frac{\xi}{2}\left(A_{1}^{2} \exp (2 \ell / \xi)-A_{1} B_{2} \exp (\ell / \xi)-A_{1} B_{1}\right) \times \\
& \left.\times \exp (-2 \ell / \xi) \int_{0}^{\infty} d y \frac{\exp (-y / \xi)}{\left(y^{2}+1\right)^{2}}\right\} .
\end{aligned}
$$

In order to determine the asymptotic behavior of the integrals in Eqs. (E40)- E42) we repeatedly integrate by parts so that

$$
\begin{gathered}
\int_{0}^{\ell-d_{w}} d y \frac{\exp (y / \xi)}{\left(y^{2}+1\right)^{2}}=\frac{\xi \exp \left(\left(\ell-d_{w}\right) / \xi\right)}{\left(\left(\ell-d_{w}\right)^{2}+1\right)^{2}}-\xi+\xi \int_{0}^{\ell-d_{w}} \frac{4 y}{\left(y^{2}+1\right)^{3}} \exp (y / \xi) \\
=\frac{\xi \exp \left(\left(\ell-d_{w}\right) / \xi\right)}{\ell^{4}}+\mathcal{O}\left(\ell^{-5} \exp (\ell / \xi)\right), \quad \ell \gg 1 \\
\int_{0}^{\ell-d_{w}} d y \frac{\exp (-y / \xi)}{\left(y^{2}+1\right)^{2}}=\int_{0}^{\infty} d y \frac{\exp (-y / \xi)}{\left(y^{2}+1\right)^{2}}-\int_{\ell-d_{w}}^{\infty} d y \frac{\exp (-y / \xi)}{\left(y^{2}+1\right)^{2}}
\end{gathered}
$$

with

$$
\begin{aligned}
\int_{\ell-d_{w}}^{\infty} d y \frac{\exp (-y / \xi)}{\left(y^{2}+1\right)^{2}} & =\frac{\xi \exp \left(-\left(\ell-d_{w}\right) / \xi\right)}{\left(\left(\ell-d_{w}\right)^{2}+1\right)^{2}}-\xi \int_{\ell}^{\infty} \frac{4 y}{\left(y^{2}+1\right)^{3}} \exp (-y / \xi) \\
& =\frac{\xi \exp \left(-\left(\ell-d_{w}\right) / \xi\right)}{\ell^{4}}+\mathcal{O}\left(\ell^{-5} \exp (-\ell / \xi)\right), \quad \ell \gg d_{w}
\end{aligned}
$$


and

$$
\begin{aligned}
\int_{d_{w}-\ell}^{\infty} d y \frac{\exp (-y / \xi)}{\left(y^{2}+1\right)^{2}} & =\frac{\xi \exp \left(\left(\ell-d_{w}\right) / \xi\right)}{\left(\left(\ell-d_{w}\right)^{2}+1\right)^{2}}-\xi \int_{d_{w}-\ell}^{\infty} \frac{4 y}{\left(y^{2}+1\right)^{3}} \exp (-y / \xi) \\
& =\frac{\xi \exp \left(\left(\ell-d_{w}\right) / \xi\right)}{\ell^{4}}+\mathcal{O}\left(\ell^{-5} \exp (\ell / \xi)\right), \quad \ell \gg d_{w} .
\end{aligned}
$$

Finally, collecting the leading terms for $\ell \rightarrow \infty$ one obtains the asymptotic behavior

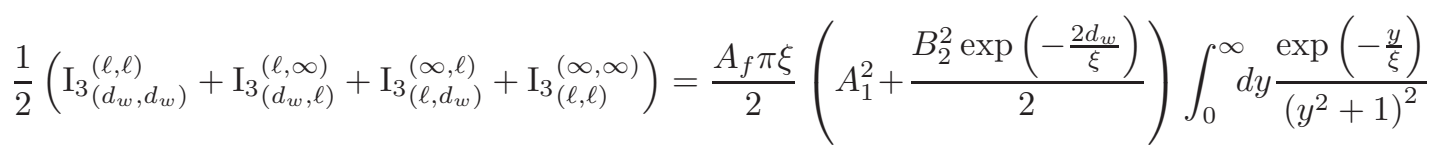

$$
\begin{aligned}
& -\frac{A_{f} \pi A_{1}^{2}}{2}\left(\frac{1}{2}-\frac{1}{2 \xi} \int_{0}^{\infty} d y \frac{\exp (-y / \xi)}{y^{2}+1}\right)+\mathcal{O}\left(\frac{1}{\ell^{4}}\right) \text {. }
\end{aligned}
$$

Inserting the results for these integrals (see Eqs. (E14), (E15), (E27), (E28), (E30), (E32), and (E49) into Eq. (E1), one obtains the effective interface potential $\omega(\ell)=\Omega_{s, l r}(\ell)-\Omega_{s, l r}(\infty)$ given by Eq. (56); the index $l r$ refers to long-ranged interactions (Sec. III).

[1] N. A. Denesyuk and J.-P. Hansen, Europhys. Lett. 63, 261 (2003).

[2] N. A. Denesyuk and J.-P. Hansen, J. Chem. Phys. 121, 3613 (2004).

[3] A. Oleksy and J.-P. Hansen, Mol. Phys. 107, 2609 (2009).

[4] A. Oleksy and J.-P. Hansen, J. Chem. Phys. 132, 204702 (2010).

[5] I. Ibagon, M. Bier, and S. Dietrich, J. Chem. Phys. 138, 214703 (2013).

[6] R. Evans, Adv. Phys. 28, 143 (1979).

[7] M. Schick, in Liquids at interfaces, edited by J. Charvolin, J. F. Joanny, and J. Zinn-Justin (North-Holland, Amsterdam, 1988), p. 415.

[8] S. Dietrich, in Phase Transitions and Critical Phenomena, edited by C. Domb and J. L. Lebowitz (Academic, London, 1988), Vol. 12, p. 1.

[9] M. Bier, A. Gambassi, and S. Dietrich, J. Chem. Phys. 137, 034504 (2012).

[10] M. Rubinstein and R. H. Colby, Polymer Physics (Oxford University Press, 2004).

[11] J. W. Cahn and J. E. Hilliard, J. Chem. Phys. 28, 258 (1958).

[12] H. Nakanishi and M. Fisher, Phys. Rev. Lett. 49, 1565 (1982).

[13] C.J.F. Böttcher, Theory of Electric Polarization (Elsevier, Amsterdam, 1973).

[14] L. Bocquet, E. Trizac, and M. Aubouy J. Chem. Phys. 117, 8138 (2002).

[15] T. Aukrust and E. H. Hauge, Phys. Rev. Lett. 54, 1814 (1985).

[16] T. Getta and S. Dietrich, Phys. Rev. E 57, 655 (1998).
[17] J. N. Israelachvili, Intermolecular and surface forces, 2nd ed. (Academic Press, 1991).

[18] J. O. Indekeu, K. Ragil, D. Bonn, D. Broseta, and J. Meunier, J. Stat. Phys. 95, 1009 (1999).

[19] J. O. Indekeu, Phys. Rev. Lett. 85, 4188 (2000).

[20] J. Piasecki and E. H. Hauge, Physica A 143, 87 (1987).

[21] J. O. Indekeu, Europhys. Lett. 10, 165 (1989).

[22] G. Langie and J. O. Indekeu, J. Phys. Condens. Matter 3, 9797 (1991).

[23] S. Dietrich and M. Schick, Phys. Rev. B 31, 4718 (1985).

[24] N. Shahidzadeh, D. Bonn, K. Ragil, D. Broseta, and J. Meunier, Phys. Rev. Lett. 80, 3992 (1998).

[25] D. E. Sullivan, Phys. Rev. B 20, 3991 (1979).

[26] S. Dietrich and M. Napiórkowski, Phys. Rev. A 43, 1861 (1991).

[27] T. Meister and H. Müller-Krumbhaar, Phys. Rev. Lett. 51, 1780 (1983).

[28] R. Lipowsky, Z. Phys. B 55, 345 (1984).

[29] R. Hołyst and A. Poniewierski, Phys. Rev. A 36, 5628 (1987).

[30] M.P. Gelfand and R. Lipowsky, Phys. Rev. B 36, 8725 (1987).

[31] A.J. Jin and M.E. Fisher, Phys. Rev. B 47, 7365 (1993).

[32] M. Iwamatsu, J. Phys.: Condens. Matter 5, 7537 (1993).

[33] A.O. Parry, C. Rascón, N.R. Bernardino, and J.M. Romero-Enrique, J. Phys.: Condens. Matter 18, 6433 (2006).

[34] A. Wójtowicz and M. Napiórkowski, J. Phys.: Condens. Matter 25, 485007 (2013). 